CERN-PPE/97-002

January 8, 1997

\title{
A Measurement of the QCD Colour Factors and a Limit on the Light Gluino
}

The ALEPH Collaboration

\begin{abstract}
Using data collected from 1992 to 1995 with the ALEPH detector at LEP, a measurement of the colour factor ratios $C_{A} / C_{F}$ and $T_{F} / C_{F}$ and the strong coupling constant $\bar{\alpha}_{s}=$ $C_{F} \alpha_{s}\left(\mathrm{M}_{\mathrm{Z}}\right) /(2 \pi)$ has been performed by fitting theoretical predictions simultaneously to the measured differential two-jet rate and angular distributions in four-jet events. The result is found to be in excellent agreement with QCD,

$$
\frac{C_{A}}{C_{F}}=2.20 \pm 0.09(\text { stat }) \pm 0.13(\text { syst }) \quad, \quad \frac{T_{F}}{C_{F}}=0.29 \pm 0.05(\text { stat }) \pm 0.06(\text { syst })
$$

Fixing $C_{A} / C_{F}$ and $T_{F} / C_{F}$ to the QCD values permits a determination of $\alpha_{s}\left(\mathrm{M}_{\mathrm{Z}}\right)$ and $n_{f}$, the number of active flavours. With this measurement the existence of a gluino with mass below $6.3 \mathrm{GeV} / c^{2}$ is excluded at $95 \%$ confidence level.
\end{abstract}

Submitted to Zeitschrift für Physik $C$ 


\section{The ALEPH Collaboration}

R. Barate, D. Buskulic, D. Decamp, P. Ghez, C. Goy, J.-P. Lees, A. Lucotte, M.-N. Minard, J.-Y. Nief, P. Odier, B. Pietrzyk

Laboratoire de Physique des Particules (LAPP), IN² $P^{3}$-CNRS, 74019 Annecy-le-Vieux Cedex, France

M.P. Casado, M. Chmeissani, P. Comas, J.M. Crespo, M. Delfino, E. Fernandez, M. Fernandez-Bosman, Ll. Garrido, ${ }^{15}$ A. Juste, M. Martinez, R. Miquel, Ll.M. Mir, S. Orteu, C. Padilla, I.C. Park, A. Pascual, J.A. Perlas, I. Riu, F. Sanchez, F. Teubert Institut de Fisica d'Altes Energies, Universitat Autonoma de Barcelona, 08193 Bellaterra (Barcelona), Spain $^{7}$

A. Colaleo, D. Creanza, M. de Palma, G. Gelao, G. Iaselli, G. Maggi, M. Maggi, N. Marinelli, S. Nuzzo, A. Ranieri, G. Raso, F. Ruggieri, G. Selvaggi, L. Silvestris, P. Tempesta, A. Tricomi, ${ }^{3}$ G. Zito Dipartimento di Fisica, INFN Sezione di Bari, 70126 Bari, Italy

X. Huang, J. Lin, Q. Ouyang, T. Wang, Y. Xie, R. Xu, S. Xue, J. Zhang, L. Zhang, W. Zhao Institute of High-Energy Physics, Academia Sinica, Beijing, The People's Republic of China ${ }^{8}$

D. Abbaneo, R. Alemany, A.O. Bazarko, ${ }^{25}$ P. Bright-Thomas, M. Cattaneo, F. Cerutti, H. Drevermann, R.W. Forty, M. Frank, R. Hagelberg, J. Harvey, P. Janot, B. Jost, E. Kneringer, J. Knobloch, I. Lehraus, G. Lutters, P. Mato, A. Minten, L. Moneta, A. Pacheco, J.-F. Pusztaszeri, F. Ranjard, P. Rensing, ${ }^{12}$ G. Rizzo, L. Rolandi, D. Schlatter, M. Schmelling, ${ }^{26}$ M. Schmitt, O. Schneider, W. Tejessy, I.R. Tomalin, H. Wachsmuth, A. Wagner European Laboratory for Particle Physics (CERN), 1211 Geneva 23, Switzerland

Z. Ajaltouni, A. Barrès, C. Boyer, A. Falvard, C. Ferdi, P. Gay, C . Guicheney, P. Henrard, J. Jousset, B. Michel, S. Monteil, J-C. Montret, D. Pallin, P. Perret, F. Podlyski, J. Proriol, P. Rosnet, J.-M. Rossignol Laboratoire de Physique Corpusculaire, Université Blaise Pascal, $I N^{2} P^{3}$-CNRS, Clermont-Ferrand, 63177 Aubière, France

T. Fearnley, J.B. Hansen, J.D. Hansen, J.R. Hansen, P.H. Hansen, B.S. Nilsson, B. Rensch, A. Wäänänen Niels Bohr Institute, 2100 Copenhagen, Denmark ${ }^{9}$

G. Daskalakis, A. Kyriakis, C. Markou, E. Simopoulou, I. Siotis, A. Vayaki, K. Zachariadou Nuclear Research Center Demokritos (NRCD), Athens, Greece

A. Blondel, G. Bonneaud, J.C. Brient, P. Bourdon, A. Rougé, M. Rumpf, A. Valassi, ${ }^{6}$ M. Verderi, H. Videau Laboratoire de Physique Nucléaire et des Hautes Energies, Ecole Polytechnique, IN ${ }^{2} P^{3}-C N R S, 91128$ Palaiseau Cedex, France

D.J. Candlin, M.I. Parsons

Department of Physics, University of Edinburgh, Edinburgh EH9 3JZ, United Kingdom ${ }^{10}$

E. Focardi, G. Parrini

Dipartimento di Fisica, Università di Firenze, INFN Sezione di Firenze, 50125 Firenze, Italy

M. Corden, C. Georgiopoulos, D.E. Jaffe

Supercomputer Computations Research Institute, Florida State University, Tallahassee, FL 323064052, USA ${ }^{13,14}$

A. Antonelli, G. Bencivenni, G. Bologna, ${ }^{4}$ F. Bossi, P. Campana, G. Capon, D. Casper, V. Chiarella, G. Felici, P. Laurelli, G. Mannocchi, ${ }^{5}$ F. Murtas, G.P. Murtas, L. Passalacqua, M. Pepe-Altarelli

Laboratori Nazionali dell'INFN (LNF-INFN), 00044 Frascati, Italy 
L. Curtis, S.J. Dorris, A.W. Halley, I.G. Knowles, J.G. Lynch, V. O'Shea, C. Raine, J.M. Scarr, K. Smith,

P. Teixeira-Dias, A.S. Thompson, E. Thomson, F. Thomson, R.M. Turnbull

Department of Physics and Astronomy, University of Glasgow, Glasgow G12 8QQ,United Kingdom ${ }^{10}$

U. Becker, C. Geweniger, G. Graefe, P. Hanke, G. Hansper, V. Hepp, E.E. Kluge, A. Putzer, M. Schmidt, J. Sommer, H. Stenzel, K. Tittel, S. Werner, M. Wunsch

Institut für Hochenergiephysik, Universität Heidelberg, 69120 Heidelberg, Fed. Rep. of Germany ${ }^{16}$

R. Beuselinck, D.M. Binnie, W. Cameron, P.J. Dornan, M. Girone, S. Goodsir, E.B. Martin, A. Moutoussi, J. Nash, J.K. Sedgbeer, A.M. Stacey, M.D. Williams

Department of Physics, Imperial College, London SW7 2BZ, United Kingdom ${ }^{10}$

G. Dissertori, P. Girtler, D. Kuhn, G. Rudolph

Institut für Experimentalphysik, Universität Innsbruck, 6020 Innsbruck, Austria ${ }^{18}$

A.P. Betteridge, C.K. Bowdery, P. Colrain, G. Crawford, A.J. Finch, F. Foster, G. Hughes, T. Sloan, M.I. Williams

Department of Physics, University of Lancaster, Lancaster LA1 4YB, United Kingdom ${ }^{10}$

A. Galla, I. Giehl, A.M. Greene, C. Hoffmann, K. Jakobs, K. Kleinknecht, G. Quast, B. Renk, E. Rohne, H.-G. Sander, P. van Gemmeren, C. Zeitnitz

Institut für Physik, Universität Mainz, 55099 Mainz, Fed. Rep. of Germany ${ }^{16}$

J.J. Aubert, C. Benchouk, A. Bonissent, G. Bujosa, D. Calvet, J. Carr, P. Coyle, C. Diaconu, F. Etienne,

N. Konstantinidis, O. Leroy, P. Payre, D. Rousseau, M. Talby, A. Sadouki, M. Thulasidas, K. Trabelsi

Centre de Physique des Particules, Faculté des Sciences de Luminy, IN ${ }^{2} P^{3}$-CNRS, 13288 Marseille,

France

M. Aleppo, F. Ragusa ${ }^{2}$

Dipartimento di Fisica, Università di Milano e INFN Sezione di Milano, 20133 Milano, Italy

R. Berlich, W. Blum, V. Büscher, H. Dietl, F. Dydak, ${ }^{2}$ G. Ganis, C. Gotzhein, H. Kroha, G. Lütjens, G. Lutz, W. Männer, H.-G. Moser, R. Richter, A. Rosado-Schlosser, S. Schael, R. Settles, H. Seywerd, R. St. Denis, H. Stenzel, W. Wiedenmann, G. Wolf

Max-Planck-Institut für Physik, Werner-Heisenberg-Institut, 80805 München, Fed. Rep. of Germany ${ }^{16}$

J. Boucrot, O. Callot, ${ }^{2}$ S. Chen, Y. Choi, ${ }^{21}$ A. Cordier, M. Davier, L. Duflot, J.-F. Grivaz, Ph. Heusse, A. Höcker, A. Jacholkowska, M. Jacquet, D.W. Kim ${ }^{19}$ F. Le Diberder, J. Lefrançois, A.-M. Lutz, I. Nikolic, H.J. Park, ${ }^{19}$

M.-H. Schune, S. Simion, J.-J. Veillet, I. Videau, D. Zerwas

Laboratoire de l'Accélérateur Linéaire, Université de Paris-Sud, $I N^{2} P^{3}$-CNRS, 91405 Orsay Cedex,

France

P. Azzurri, G. Bagliesi, G. Batignani, S. Bettarini, C. Bozzi, G. Calderini, M. Carpinelli, M.A. Ciocci, V. Ciulli, R. Dell'Orso, R. Fantechi, I. Ferrante, L. Foà, ${ }^{1}$ F. Forti, A. Giassi, M.A. Giorgi, A. Gregorio, F. Ligabue, A. Lusiani, P.S. Marrocchesi, A. Messineo, F. Palla, G. Sanguinetti, A. Sciabà, P. Spagnolo, J. Steinberger, R. Tenchini, G. Tonelli, ${ }^{24}$ C. Vannini, A. Venturi, P.G. Verdini

Dipartimento di Fisica dell'Università, INFN Sezione di Pisa, e Scuola Normale Superiore, 56010 Pisa, Italy

G.A. Blair, L.M. Bryant, J.T. Chambers, Y. Gao, M.G. Green, T. Medcalf, P. Perrodo, J.A. Strong, J.H. von Wimmersperg-Toeller

Department of Physics, Royal Holloway \& Bedford New College, University of London, Surrey TW20 OEX, United Kingdom ${ }^{10}$

D.R. Botterill, R.W. Clifft, T.R. Edgecock, S. Haywood, P. Maley, P.R. Norton, J.C. Thompson, A.E. Wright Particle Physics Dept., Rutherford Appleton Laboratory, Chilton, Didcot, Oxon OX11 OQX, United Kingdom ${ }^{10}$

B. Bloch-Devaux, P. Colas, S. Emery, W. Kozanecki, E. Lançon, M.C. Lemaire, E. Locci, P. Perez, J. Rander, J.-F. Renardy, A. Roussarie, J.-P. Schuller, J. Schwindling, A. Trabelsi, B. Vallage

CEA, DAPNIA/Service de Physique des Particules, CE-Saclay, 91191 Gif-sur-Yvette Cedex, France ${ }^{17}$ 
S.N. Black, J.H. Dann, R.P. Johnson, H.Y. Kim, A.M. Litke, M.A. McNeil, G. Taylor

Institute for Particle Physics, University of California at Santa Cruz, Santa Cruz, CA 95064, USA ${ }^{22}$

C.N. Booth, R. Boswell, C.A.J. Brew, S. Cartwright, F. Combley, M.S. Kelly, M. Lehto, W.M. Newton, J. Reeve, L.F. Thompson

Department of Physics, University of Sheffield, Sheffield S3 7RH, United Kingdom ${ }^{10}$

A. Böhrer, S. Brandt, G. Cowan, C. Grupen, P. Saraiva, L. Smolik, F. Stephan

Fachbereich Physik, Universität Siegen, 57068 Siegen, Fed. Rep. of Germany ${ }^{16}$

M. Apollonio, L. Bosisio, R. Della Marina, G. Giannini, B. Gobbo, G. Musolino

Dipartimento di Fisica, Università di Trieste e INFN Sezione di Trieste, 34127 Trieste, Italy

J. Rothberg, S. Wasserbaech

Experimental Elementary Particle Physics, University of Washington, WA 98195 Seattle, U.S.A.

S.R. Armstrong, P. Elmer, Z. Feng, ${ }^{20}$ D.P.S. Ferguson, Y.S. Gao, ${ }^{23}$ S. González, J. Grahl, T.C. Greening, O.J. Hayes, H. Hu, P.A. McNamara III, J.M. Nachtman, W. Orejudos, Y.B. Pan, Y. Saadi, I.J. Scott, J. Walsh, Sau Lan Wu, X. Wu, J.M. Yamartino, M. Zheng, G. Zobernig

Department of Physics, University of Wisconsin, Madison, WI 53706, USA ${ }^{11}$

\footnotetext{
${ }^{1}$ Now at CERN, 1211 Geneva 23, Switzerland.

${ }^{2}$ Also at CERN, 1211 Geneva 23, Switzerland.

${ }^{3}$ Also at Dipartimento di Fisica, INFN, Sezione di Catania, Catania, Italy.

${ }^{4}$ Also Istituto di Fisica Generale, Università di Torino, Torino, Italy.

${ }^{5}$ Also Istituto di Cosmo-Geofisica del C.N.R., Torino, Italy.

${ }^{6}$ Supported by the Commission of the European Communities, contract ERBCHBICT941234.

${ }^{7}$ Supported by CICYT, Spain.

${ }^{8}$ Supported by the National Science Foundation of China.

${ }^{9}$ Supported by the Danish Natural Science Research Council.

${ }^{10}$ Supported by the UK Particle Physics and Astronomy Research Council.

${ }^{11}$ Supported by the US Department of Energy, grant DE-FG0295-ER40896.

${ }^{12}$ Now at Dragon Systems, Newton, MA 02160, U.S.A.

${ }^{13}$ Supported by the US Department of Energy, contract DE-FG05-92ER40742.

${ }^{14}$ Supported by the US Department of Energy, contract DE-FC05-85ER250000.

${ }^{15}$ Permanent address: Universitat de Barcelona, 08208 Barcelona, Spain.

${ }^{16}$ Supported by the Bundesministerium für Bildung, Wissenschaft, Forschung und Technologie, Fed. Rep. of Germany.

${ }^{17}$ Supported by the Direction des Sciences de la Matière, C.E.A.

${ }^{18}$ Supported by Fonds zur Förderung der wissenschaftlichen Forschung, Austria.

${ }^{19}$ Permanent address: Kangnung National University, Kangnung, Korea.

${ }^{20}$ Now at The Johns Hopkins University, Baltimore, MD 21218, U.S.A.

${ }^{21}$ Permanent address: Sung Kyun Kwan University, Suwon, Korea.

${ }^{22}$ Supported by the US Department of Energy, grant DE-FG03-92ER40689.

${ }^{23}$ Now at Harvard University, Cambridge, MA 02138, U.S.A.

${ }^{24}$ Also at Istituto di Matematica e Fisica, Università di Sassari, Sassari, Italy.

${ }^{25}$ Now at Princeton University, Princeton, NJ 08544, U.S.A.

${ }^{26}$ Now at Max-Planck-Institut für Kernphysik, Heidelberg, Germany
} 


\section{Introduction}

Quantum Chromodynamics (QCD), the theory of strong interactions, has been found to describe successfully many aspects of the dynamics of quarks and gluons, despite the lack of knowledge about the non-perturbative phase in which quarks and gluons form colour neutral hadrons. The success is based on the fact that the effective running coupling of QCD decreases with increasing energy, a property which improves the reliability of perturbative calculations, and that the impact of non-perturbative effects becomes less important at the same time, especially when looking at appropriately defined quantities such as jets. For this purpose LEP I is a beautiful laboratory, having delivered to ALEPH about 5 million $e^{+} e^{-}$annihilations into hadronic final states. The purely leptonic initial state facilitates the theoretical calculations, and the high energy $\left(s=\mathrm{M}_{\mathrm{Z}}^{2}\right)$ brings the directions and energies of hadronic jets into close correspondence with those of the underlying partons.

A very stringent test of QCD would be a simultaneous measurement of the strong coupling constant $\alpha_{s}$ and the colour factors, as the former is the only free parameter of the theory, and the latter show whether the dynamics is indeed described by an unbroken SU(3) symmetry. To achieve this, cross sections have to be measured for which perturbative predictions exist at least up to $\mathcal{O}\left(\alpha_{s}^{2}\right)$, since only starting with this order does a functional dependence on the colour factors appear. In this analysis two types of variables have been measured. For the first type the predictions start at $\mathcal{O}\left(\alpha_{s}\right)$, giving information on $\alpha_{s}\left(\mathrm{M}_{\mathrm{Z}}\right)$ and on the colour factors via the running of $\alpha_{s}$. For the second type the predictions start only at $\mathcal{O}\left(\alpha_{s}^{2}\right)$, but additional sensitivity to the colour factors is obtained. Under the assumption that $\mathrm{SU}(3)$ is the underlying gauge group, a measurement of the colour factors can be converted into a measurement of the number of active flavours. This number could be altered from its expectation of five by new physics, such as the existence of a very light gluino.

The paper is organized as follows : In the next section the theoretical framework is described in detail, then the strategy of the analysis is explained. In Section 4 the data analysis, correction and fit procedure are summarized and the fit results are presented. Section 5 contains a detailed breakdown of the systematic checks which have been performed, followed by a discussion of the results. The conclusions are given in Section 6 .

\section{Theoretical Framework}

QCD is a gauge theory with $\mathrm{SU}(3)$ as underlying gauge group. For a general gauge theory with a simple Lie group of dimension $N_{C}$, the couplings of the fermion fields to the gauge fields and the self-interactions in the non-abelian case are determined by the coupling constant and the Casimir operators of the gauge group. Measuring the eigenvalues of these operators, called colour factors, probes the underlying structure of the theory in a gauge invariant way. Considering the case where $N_{F}$ and $N_{A}$ are the dimensions of the fundamental and adjoint representations of the gauge group with structure constants $f^{a b c}$ and generators $T_{i j}^{a}$, the following relations hold :

$$
\sum_{a=1}^{N_{A}}\left(T^{a} T^{\dagger a}\right)_{i j}=\delta_{i j} C_{F} \quad, \quad \sum_{a, b=1}^{N_{A}} f^{a b c} f^{* a b d}=\delta^{c d} C_{A} \quad, \quad \sum_{i, j=1}^{N_{F}} T_{i j}^{a} T_{j i}^{\dagger b}=\delta^{a b} T_{F}
$$


where $a, b, \ldots(i, j, \ldots)$ represent gauge (fermion) field indices and $C_{F}, C_{A}$ and $T_{F}$ are the colour factors. For $\mathrm{SU}\left(N_{C}\right)$ it is found

$$
C_{A}=N_{C} \quad, \quad C_{F}=\frac{N_{C}^{2}-1}{2 N_{C}} \quad, \quad T_{F}=1 / 2 .
$$

For QCD $N_{C}=3$, hence $C_{A}=3$ and $C_{F}=4 / 3$. The first two expressions in Eq. (1) define the colour factors $C_{F}$ and $C_{A}$, which appear when calculating matrix elements related to the emission of a gauge boson by a fermion and the splitting of one gauge boson into two gauge bosons, respectively. The last expression in Eq. (1) represents a normalization of the generators and governs the rate of gauge boson splitting into fermions. These relations are illustrated in Fig. 1. Summing over all indices in the defining equations for $C_{F}$ and $T_{F}$, a connection is found between the dimensionality of the fundamental and adjoint representations, namely $N_{F} C_{F}=N_{A} T_{F}$.

In this paper two types of variables have been considered, which will be called first order and second order variables. First order variables are quantities for which the perturbative prediction starts at $\mathcal{O}\left(\alpha_{s}\right)$. Examples are event-shape distributions such as thrust, jet masses, jet broadenings or the differential two-jet rate. For a general event-shape distribution $y$, which vanishes in the limit of perfect two-jet topologies, the differential cross section can be written as (the symbols being defined in the following):

$$
\frac{1}{\sigma_{t o t}} \frac{d \sigma}{d y}=\bar{\alpha}_{s}\left(\mu^{2}\right) A(y)+\bar{\alpha}_{s}^{2}\left(\mu^{2}\right)\left(B(y)+b_{0} A(y) \ln \frac{\mu^{2}}{s}\right)+\mathcal{O}\left(\bar{\alpha}_{s}^{3}\right)
$$

Here $\sigma_{\text {tot }}$ is the total hadronic cross section, and the following redefinition of the running coupling constant has been adopted :

$$
\frac{C_{F} \alpha_{s}\left(\mu^{2}\right)}{2 \pi}=\bar{\alpha}_{s}\left(\mu^{2}\right)=\frac{\bar{\alpha}_{s}(s)}{w}\left(1-\frac{b_{1}}{b_{0}} \frac{\bar{\alpha}_{s}(s)}{w} \ln w\right) \quad, \quad w=1+b_{0} \bar{\alpha}_{s}(s) \ln \frac{\mu^{2}}{s} .
$$

Defining

$$
f_{A}=\frac{C_{A}}{C_{F}} \quad, \quad f_{T}=n_{f} \frac{T_{F}}{C_{F}},
$$

with $n_{f}$ being the number of active flavours, the two coefficients $b_{0}$ and $b_{1}$ are

$$
b_{0}=\frac{11}{6} f_{A}-\frac{2}{3} f_{T} \quad \text { and } \quad b_{1}=\frac{17}{6} f_{A}^{2}-\left(\frac{5}{3} f_{A}+1\right) f_{T} .
$$

When talking about colour factors in the following, actually the colour factor ratios $C_{A} / C_{F}$ and $T_{F} / C_{F}$ are meant.

The coefficient functions $A(y)$ and $B(y)$ are obtained by integrating the fully differential EllisRoss-Terrano (ERT) matrix elements [1]. Whereas $A(y)$, which results from the integration over the matrix elements for single gluon bremsstrahlung off quarks, is colour factor independent, $B(y)$ can be decomposed into

$$
B(y)=B_{F}(y)+f_{A} B_{A}(y)+f_{T} B_{T}(y) .
$$

The function $B_{F}(y)$ gets contributions from double gluon bremsstrahlung, which occurs at a rate proportional to $C_{F}^{2}$, the function $B_{A}(y)$ accounts mainly for processes with the triple gluon 
coupling and $B_{T}(y)$ for processes with gluon splitting into quark pairs. From this it becomes clear that information on colour factors enters only in next-to-leading order via the coefficient function $B(y)$. However, additional dependence at $\mathcal{O}\left(\bar{\alpha}_{s}^{3}\right)$ and higher orders enters through the running coupling, mainly via $b_{0}$, if the renormalization scale $\mu^{2}$ is chosen to be different from the hard scale $s$. For event-shape variables it has been found $[2,3]$ that a rather small scale $\mu^{2} \ll s$ has to be used in order to achieve a good description of the data. In this case missing higher orders, which appear to be important, are mimicked by terms generated by the expansion of the running coupling.

For several event-shape variables it is possible to resum the leading and next-to-leading logarithms $\ln y$ in all orders of $\alpha_{s}$ ([4] and references therein), restoring the hard scale for $\mu^{2}$ as the natural one. In those cases a function $R(x)$ is added to the expression in Eq. (3), where $x=b_{0} \bar{\alpha}_{s}\left(\mu^{2}\right) \ln y$, thus again $b_{0}$ enters in connection with the leading terms, which introduces a high correlation between the estimates of $f_{A}$ and $f_{T}$. Summarizing it can be stated that first order variables are suited for measuring $\alpha_{s}\left(\mathrm{M}_{\mathrm{Z}}\right)$ and a function of $f_{A}$ and $f_{T}$, namely $b_{0}$.

It is worth noting that information on $n_{f}$ enters via $f_{T}$. This is the number of fermions which give contributions to loop corrections and to gluon splitting processes. At LEP $n_{f}=5$ is expected, but if additional fermionic degrees of freedom with colour charge exist, then a sizeable effect on $b_{0}$ should be observed. A possible candidate for such additional fermions would be a very light gluino in the mass range $m_{\tilde{g}} \lesssim 1.5 \mathrm{GeV} / c^{2}$, which is predicted by particular SUSY models and not yet excluded unambiguously by experiment [5].

Uncorrelated information on $f_{A}$ and $f_{T}$ can be obtained from second order variables, for which the perturbative expansion starts only at $\mathcal{O}\left(\alpha_{s}^{2}\right)$, like thrust-minor, light jet mass or angular variables in four-jet events. Here the cross section is given by

$$
\frac{1}{\sigma_{t o t}} \frac{d \sigma}{d y}=N\left[D_{F}(y)+f_{A} D_{A}(y)+f_{T} D_{T}(y)\right]
$$

with coefficient functions $D_{*}(y)(*=F, A, T)$ again found by integration of the ERT matrix elements for processes which have been described in the case of the functions $B_{*}(y)$. Such variables have the advantage that colour factor information already enters in leading order, but normally they are not used to measure $\alpha_{s}$, as only Born level calculations are available. This fact is expressed in Eq. (8) by the replacement of the strong coupling by an effective coupling or normalization constant $N$, which typically is fitted together with $f_{A}$ and $f_{T}$. Also in this case a light gluino would alter the cross section.

The idea of the measurement described in this paper is to combine the information on $\bar{\alpha}_{s}\left(\mathrm{M}_{\mathrm{Z}}\right)$, $f_{A}$ and $f_{T}$ obtained from first and second order variables, leading to precise determinations of all three variables. Assuming QCD and fitting only $\alpha_{s}\left(\mathrm{M}_{\mathrm{Z}}\right)$ and $f_{T}$, it is possible to extract knowledge on $n_{f}$. Thus the measurement represents an indirect search for a very light gluino, which is rather independent of the decay properties of such an object, as the main sensitivity stems from loop effects. Finally fixing $n_{f}$ and using only a first order variable for the fit gives a determination of $\alpha_{s}\left(\mathrm{M}_{\mathrm{Z}}\right)$. This serves as a valuable cross check of the results obtained in the more general analysis. 


\section{Analysis Strategy}

The distributions used in this analysis are the differential two-jet rate as first order variable and the following four-jet angular distributions [6] as second order variables. The numbering $i=1 \ldots 4$ of the jet momenta $\mathbf{p}_{i}$ corresponds to energy-ordered four-jet configurations $\left(E_{1}>E_{2}>E_{3}>E_{4}\right)$ :

- the Bengtsson-Zerwas angle : $\chi_{B Z}=\measuredangle\left[\left(\mathbf{p}_{1} \times \mathbf{p}_{2}\right),\left(\mathbf{p}_{3} \times \mathbf{p}_{4}\right)\right]$

- the Körner-Schierholz-Willrodt angle : $\Phi_{K S W}=1 / 2\left\{\measuredangle\left[\left(\mathbf{p}_{1} \times \mathbf{p}_{4}\right),\left(\mathbf{p}_{2} \times \mathbf{p}_{3}\right)\right]+\measuredangle\left[\left(\mathbf{p}_{1} \times \mathbf{p}_{3}\right),\left(\mathbf{p}_{2} \times \mathbf{p}_{4}\right)\right]\right\}$

- the (modified) Nachtmann-Reiter angle : $\theta_{N R}^{*}=\measuredangle\left[\left(\mathbf{p}_{1}-\mathbf{p}_{2}\right),\left(\mathbf{p}_{3}-\mathbf{p}_{4}\right)\right]$

- the angle between the two lowest energetic jets : $\alpha_{34}=\measuredangle\left[\mathbf{p}_{3}, \mathbf{p}_{4}\right]$

Jets are found by applying the Durham cluster algorithm [7, 8, 9], with the metric

$$
y_{i j}=\frac{2 \min \left(E_{i}^{2}, E_{j}^{2}\right)}{E_{v i s}^{2}}\left(1-\cos \Theta_{i j}\right)
$$

for particles $i, j$, and the E0 recombination scheme, i.e., those particles with the smallest $y_{i j}$ are clustered together to form a new pseudo-particle with four-momentum

$$
E_{n e w}=E_{i}+E_{j} \quad, \quad \mathbf{p}_{n e w}=\frac{E_{i}+E_{j}}{\left|\mathbf{p}_{i}+\mathbf{p}_{j}\right|}\left(\mathbf{p}_{i}+\mathbf{p}_{j}\right)
$$

hence massless jets are formed. The clustering procedure is repeated until four jets are left, then the event is used for the analysis of the angles defined above if $\min _{i, j=1,4} y_{i j}>y_{\text {cut }}$, with $y_{\text {cut }}=0.008$. This cut value represents a compromise between high statistics and good separation of the four jets. The clustering is continued to end up with three jets, independently if the $y_{\text {cut }}$ criterion for four-jets is fulfilled or not. Then $y_{3}=\min _{i, j=1,3} y_{i j}$ is calculated, which gives the distribution in $-\ln y_{3}$, called differential two-jet rate, i.e., $y_{3}$ is the resolution parameter where the event undergoes the transition from a three-jet to a two-jet event.

The choice of these variables is motivated by the following considerations : The theoretical prediction for $-\ln y_{3}$ is known up to $\mathcal{O}\left(\alpha_{s}{ }^{2}\right)$. In addition both leading and next-to-leading logarithms have been resummed in all orders of $\alpha_{s}[8,10]$. This variable is further known to suffer less from systematic uncertainties due to hadronization than other event-shape distributions [11]. The four-jet angular distributions have already been used in previous measurements and proven to be sensitive to the colour factors $[12,13,14]$.

The measured distributions of the variables are binned into 20 bins each and put together to form a vector $\mathbf{D}$,

$$
D_{1 \ldots 100}=\left(V_{1 \ldots 20}, W_{1 \ldots 20}, X_{1 \ldots 20}, Y_{1 \ldots 20}, Z_{1 \ldots 20}\right) \quad,
$$

where $V, W, X, Y$ stand for the four angular variables and $Z$ for the differential two-jet rate. A covariance matrix $\sigma_{i j}^{D}$ is calculated to take into account the statistical error, correlations between bins of a single distribution, and correlations between bins of different distributions. 
The predictions at parton level, $\mathbf{P}$, which are functions of $\bar{\alpha}_{s}, f_{A}$ and $f_{T}$, are corrected to give predictions $\mathbf{T}$ at detector level according to

$$
\mathbf{T}=M^{D} \cdot R \cdot M^{H} \cdot \mathbf{P}
$$

$\mathbf{T}$ being a vector of the same dimension as $\mathbf{D}$. The matrices $M_{i j}^{H}\left(M_{i j}^{D}\right)$ are the transition probabilities that an event with an entry in bin $j$ at parton (hadron) level gives an entry in bin $i$ at hadron (detector) level. The ratio $R_{i}=(\mathrm{ISR}+\mathrm{FSR})_{i} /(\text { no ISR, no FSR })_{i}$ corrects for effects of initial and final state radiation (ISR,FSR), which are not included in the perturbative calculations. To account for finite Monte Carlo statistics, statistical errors are propagated into a final covariance matrix $\sigma_{i j}^{T}$.

Having prepared all the necessary input, the following function is computed and minimized with respect to $\bar{\alpha}_{s}, f_{A}$ and $f_{T}$ :

$$
\chi^{2}=\sum_{i, j \in \text { fit range }} \delta_{i} \sigma_{i j}^{-1} \delta_{j} \quad, \quad \delta_{i}=D_{i}-T_{i} \quad, \quad \sigma_{i j}=\sigma_{i j}^{D}+\sigma_{i j}^{T} .
$$

This method of least squares was preferred over the method of maximum likelihood adopted in a previous measurement by ALEPH [15], as it is more convenient in the case of very high statistics.

\section{Analysis Procedure and Results}

\subsection{The ALEPH Detector}

A detailed description of the ALEPH detector can be found in Ref. [16] and of its performance in Ref. [17]. Charged particles are detected in the central part of the detector consisting of a vertex detector, a cylindrical drift chamber and a large time projection chamber, which together measure up to 31 coordinates along the charged particle trajectories. A $1.5 \mathrm{~T}$ axial magnetic field is provided by a superconducting solenoidal coil. A $1 / p_{\perp}$ resolution of $6 \times 10^{-4}(\mathrm{GeV} / c)^{-1}$ is achieved for tracks with a polar angle of $|\cos \theta|<0.8$.

Electrons and photons are identified in the electromagnetic calorimeter by their characteristic longitudinal and transverse shower developments. The calorimeter, a lead/wire-plane sampling device with fine read-out segmentation and total thickness of 22 radiation lengths at normal incidence, provides a relative energy resolution of $0.18 / \sqrt{E}(E$ in $\mathrm{GeV})$.

Muons are identified by the hadron calorimeter, a $1.2 \mathrm{~m}$ thick yoke instrumented with 23 layers of streamer tubes, together with two surrounding layers of muon chambers. In association with the electromagnetic calorimeter, the hadron calorimeter also provides a measurement of the energy of charged and neutral hadrons with a relative resolution of $0.80 / \sqrt{E}(E$ in $\mathrm{GeV})$.

Combining the information of all subdetectors, an energy-flow algorithm [17] provides a measurement of the total energy and a list of charged and neutral reconstructed objects, called energy-flow objects, with measured momentum vectors and information on particle type. 


\subsection{Data Analysis}

In this analysis data taken at the $\mathrm{Z}$ peak from 1992 to 1995 are used. First a hadronic event selection is applied. Charged particle tracks are selected that have at least four measured space coordinates from the time projection chamber, a polar angle in the range $20^{\circ}<\theta<160^{\circ}$, and a transverse momentum with respect to the beam direction of $p_{\perp}>0.2 \mathrm{GeV} / c$. In addition, the closest radial distance of approach of the extrapolated track to the beam axis, $d_{0}$, is required to be less than $2 \mathrm{~cm}$, and the $z$-coordinate of the point of closest radial approach, $z_{0}$, is required to be less than $5 \mathrm{~cm}$. Using these selected charged particle tracks, the sphericity axis and the

total charged energy $E_{c h}=\sum_{i} \sqrt{p_{i}^{2}+m_{\pi}^{2}}$ are computed. Neutral energy-flow objects are kept if their energy exceeds $0.8 \mathrm{GeV}$ and if their polar angle with respect to the beam axis is in the range $11.5^{\circ}<\theta_{\text {neu }}<168.5^{\circ}$.

Events are selected that have at least five charged particle tracks, $E_{c h}>15 \mathrm{GeV}$, the polar angle of the sphericity axis in the range $35^{\circ}<\theta_{s p h}<145^{\circ}$, and $N_{t o t} \geq 15$, where $N_{\text {tot }}$ is the sum of selected charged and neutral objects. The cut on the sphericity axis ensures that the event is well contained within the detector, and the final cut on the total multiplicity reduces remaining backgrounds from $\gamma \gamma$ and $\tau^{+} \tau^{-}$events to a negligible level. With a selection efficiency of approximately $78 \%$, a sample of 2.7 million hadronic events remains, which are further analysed.

Objects are clustered according to the algorithm described in the previous section until four jets are left. The energies of these jets are subsequently rescaled by imposing total energy-momentum conservation with the assumption that the four jet directions are perfectly measured. The Durham metric $y_{i j}$ is then recalculated and the event is taken as a four-jet event if $y_{\min }>0.008$. This results in a total number of 0.17 million events for which the angular variables are computed. Rescaling of jet energies is applied to improve the resolution. It also reduces considerably systematic uncertainties when repeating the analysis with different selection criteria, e.g., using charged particle tracks only.

The differential two-jet rate and the covariance matrix are finally determined. Correlations between bins of different distributions are found to be small, typically below $10 \%$. Only near the phase space limits can larger values be observed. These regions, however, are excluded in the later fit.

\subsection{Theoretical Predictions}

The Monte Carlo program EVENT [18] has been used to integrate the second order QCD ERT matrix elements [1] in order to calculate the coefficient functions $A(y), B_{*}(y), D_{*}(y)$ defined in Section 2. The program has been modified to allow the coefficients of each colour factor to be obtained separately. In total $4.3 \times 10^{8}$ events were generated.

For the differential two-jet rate, a resummation of leading and next-to-leading logarithms has been performed $[8,10]$. However, the combination of the fixed order calculation with the resummation part is ambiguous, and two schemes have been proposed [11], the so-called $R$ matching and $\log R$ matching schemes. As long as no full third order calculation is available, there are no firm theoretical grounds to prefer one over the other, and thus fits and systematic studies have been performed considering both of them. An additional theoretical ambiguity enters 
via the choice of the renormalization scale $\mu^{2}$. It has been decided to perform the nominal analysis at a scale which minimizes the overall $\chi^{2}$ of the fit, which is not necessarily the same for the two matching schemes.

\subsubsection{Correction for Hadronization Effects}

To correct the purely partonic cross sections for effects of hadronization, the transition probabilities $M_{i j}^{H}$ have been estimated using two different Monte Carlo models. In the case of the differential two-jet rate JETSET 7.405 [19] has been employed, which is based on the parton shower approach plus subsequent string fragmentation. The model parameters are taken from Ref. [20], with the exception that final state radiation is not included in the simulation. Four million events have been generated and analysed.

For the four-jet angular variables a different model has been adopted. Here the corrections are computed using HERWIG 5.9A, which is an extension of HERWIG 5.9 [21]. The process $e^{+} e^{-} \rightarrow 4$ partons has been added, where the partonic configurations are generated according to the ERT matrix elements. Afterwards a parton shower is started from each of the four partons with subsequent cluster fragmentation. This model has the advantage that it generates more final states with four well separated jets than the simple parton shower as implemented in JETSET or HERWIG, thus covering more efficiently the full four-parton phase space. In addition, since higher order effects are simulated by the parton shower which follows the hard process generation, the hadronization corrections are expected to be less model dependent. Whereas in the matrix element option in JETSET the hadronization step in the simulation has to recover missing higher orders, since a maximum of four partons are generated, the hadronization step in HERWIG 5.9A should simulate purely hadronization effects. Indeed one finds a drastic increase in fit quality (overall $\chi^{2}$ and good description of the data by the fit outside the fit range) when using HERWIG 5.9A compared to the matrix element option in JETSET 7.405. A further shortcoming of the latter model is the minimal intrinsic $y_{\text {cut }}$ (Jade type) of 0.01 , which is rather close to the cut value of the analysis, whereas in HERWIG 5.9A this value can be chosen well below 0.008 , which corresponds to a relative transverse momentum of approximately $8.1 \mathrm{GeV} / c$.

Three million events were generated using HERWIG 5.9A with parameters for the cluster fragmentation taken from Ref. [20], which are tuned parameters for HERWIG 5.8. Despite differences between the two versions of the program, no model fit was needed for HERWIG 5.9A, since a good fit quality was already obtained with the tuned parameters for HERWIG 5.8. This obvious insensitivity to the details of the hadronization process is a further argument in favour of HERWIG 5.9A. The matrices thus found show entries well concentrated along the diagonal.

\subsubsection{Corrections for Initial and Final State Radiation}

Initial state radiation (ISR) is known to have only a small effect on event shapes at the Z peak. Also corrections to the distributions considered here caused by photon radiation from quarks (FSR) are expected to be negligible. Nevertheless they have been estimated by generating five million events with and without ISR and FSR, taking an event generator based on DYMU [22] and JETSET with significantly extended decay properties of heavy flavours. Then bin-by-bin 
correction factors were calculated. As anticipated, these factors are found to be close to unity over almost all the phase space.

\subsubsection{Corrections for Detector Effects}

The final step in the folding procedure consists in correcting the theoretical distributions found at hadron level for effects of detector acceptance and resolution. For this purpose again events have been generated with the generator based on DYMU and JETSET and subsequently passed through the full ALEPH detector simulation. A total of 4 million generated and 3 million accepted events, giving 0.2 million four-jet events at the detector level, have been analysed at detector and hadron level in order to find the transition matrices $M_{i j}^{D}$. The fully simulated events at the detector level were analysed in exactly the same manner as the data, and again rather diagonal matrices with reasonably small smearing are found. The full detector simulation allows only limited Monte Carlo statistics, which has to be included in the evaluation of the final error, as described in Section 3.

Figure 2 gives a comparison of the full simulation with the data for the five distributions of interest. For all cases clear discrepancies can be observed, which may possibly originate from the parton level simulation (JETSET parton shower), which might not be appropriate for the description of hard three-jet (low $-\ln y_{3}$ region) and four-jet configurations. However, these discrepancies are not of direct consequence for the analysis, since only the transition from hadron to detector level, i.e., pure detector effects, are of interest, and the folding procedure is arranged to have as little model dependence as possible. The good fit quality (see following sections) indicates that this has been achieved.

\subsection{Fit Procedure}

Three types of $\chi^{2}$ fits were performed with MINUIT [23] :

- Fit A :

Using all five distributions, the variables $\bar{\alpha}_{s}\left(\mathrm{M}_{\mathrm{Z}}\right), f_{A}$ and $f_{T}$ are determined by fitting Eqs. (3) (plus resummation of large logarithms) and (8) to the $-\ln y_{3}$ and angular variables, respectively, over the fit ranges $0.1 \leq|\cos \phi| \leq 0.9$ for $\phi=\chi_{B Z}$ and $\phi=\theta_{N R}^{*},-0.8 \leq$ $\cos \phi \leq 0.8$ for $\phi=\Phi_{K S W}$ and $\phi=\alpha_{34}$, and $1.4 \leq-\ln y_{3} \leq 4.4$. The ranges were chosen so that the corrections were well behaved.

- Fit B :

Taking the same distributions and fit ranges as above, $\alpha_{s}\left(\mathrm{M}_{\mathrm{Z}}\right)$ and $f_{T}$ are determined, from which $n_{f}$ is extracted subsequently by assuming QCD, i.e., $C_{A} / C_{F}=2.25, T_{F} / C_{F}=0.375$.

- Fit C :

Fitting only the differential two-jet rate over the range $1.4 \leq-\ln y_{3} \leq 3.2$ and assuming QCD for the colour factors and $n_{f}=5, \alpha_{s}\left(\mathrm{M}_{\mathrm{Z}}\right)$ is extracted.

In the case of four-jet variables entering the fit, the four-jet rates contributing to the fit range are also fitted, as the numbers of four-jet events in these ranges are not necessarily the same. This increases the numerical complexity, but is of no physics relevance. 


\subsection{Fit Results}

The results for Fit A, Fit B and Fit C for both matching schemes are listed in Table 1. The errors and correlations $\rho_{*}$ are statistical only. A $\chi^{2}$ per degree of freedom close to unity is found in all cases. For Fit A a high statistical correlation between $f_{A}$ and $f_{T}$ is observed, which can be traced back to the functional dependence via $b_{0}$, as explained in Section 2. The obvious discrepancies between the two matching schemes are taken into account in the final systematic error. A graphical representation of the fit results is shown in Fig. 3.

\begin{tabular}{|c|c|c|}
\hline Fit A & $\log \mathrm{R}, \ln f=-1.10$ & $\mathrm{R}, \ln f=0.00$ \\
\hline $\bar{\alpha}_{s}\left(\mathrm{M}_{\mathrm{Z}}\right)$ & $0.0244 \pm 0.0003$ & $0.0249 \pm 0.0003$ \\
\hline$f_{A}$ & 2.31 & 2.23 \\
\hline$f_{T}$ & 1.48 & 1.78 \\
\hline$\rho_{\bar{\alpha}_{s}, f_{A}}$ & 0.48 & 0.41 \\
\hline$\rho_{\bar{\alpha}_{s}, f_{T}}$ & 0.75 & 0.72 \\
\hline$\rho_{f_{A}, f_{T}}$ & 0.94 & 0.93 \\
\hline$\chi^{2} / N_{d o f}$ & $78.14 / 72$ & $81.60 / 72$ \\
\hline Fit B & $\log \mathrm{R}, \ln f=-1.30$ & $\mathrm{R}, \ln f=0.00$ \\
\hline$\alpha_{s}\left(\mathrm{M}_{\mathrm{Z}}\right)$ & \multirow{2}{*}{$\begin{array}{ccc}0.1154 & \pm & 0.0011 \\
3.68 & \pm & 0.24\end{array}$} & \multirow{2}{*}{$\begin{array}{ccc}0.1175 & \pm & 0.0012 \\
4.88 & \pm & 0.29\end{array}$} \\
\hline$n_{f}$ & & \\
\hline$\rho_{\alpha_{s}, n_{f}}$ & 0.99 & 0.99 \\
\hline$\chi^{2} / N_{d o f}$ & $78.48 / 73$ & $81.63 / 73$ \\
\hline Fit $\mathrm{C}$ & $\log \mathrm{R}, \ln f=-1.76$ & $\mathrm{R}, \ln f=-0.25$ \\
\hline$\alpha_{s}\left(\mathrm{M}_{\mathrm{Z}}\right)$ & \multirow{2}{*}{$\begin{array}{c}0.1220 \pm 0.0002 \\
6.86 / 8\end{array}$} & $0.1176 \pm 0.0002$ \\
\hline$\chi^{2} / N_{d o f}$ & & $5 . \overline{41 / 8}$ \\
\hline
\end{tabular}

Table 1: Results of Fit A, Fit B and Fit C $\left(\ln f=\ln \mu^{2} / s\right)$.

\section{$5 \quad$ Study of Systematic Uncertainties}

The special choice of an analysis procedure, such as event and particle selection criteria or usage of a certain Monte Carlo (MC) model for hadronization corrections, could introduce biases into the results. These biases typically are estimated by varying cuts and MC models. However, the number and range of such variations, together with the subsequent definition of a systematic error when changes of fit results appear, are rather arbitrary and differ between analyses and experiments. Here a scheme is presented which tries to reduce somewhat this arbitrariness, and which in principle is applicable to a wide range of analyses and to measurements of any number of observables.

\subsection{Definition of the Scheme}

The scheme exploits Bayesian points of view in order to assess systematic uncertainties. The main decision criterion is the quality of the fit when using a particular analysis chain, in our case the 
overall $\chi^{2}$. The Bayesian idea is that a priori all models can be considered equally well suited for usage in the analysis, but from a bad $\chi^{2}$ it is deduced that the a posteriori probability of such a model is low, and therefore this model should get a small weight when estimating the actual systematic error. In accordance to the determination of the statistical error, the systematic error corresponds to the increase in $\chi^{2}$ by one.

For example, a measurement of two quantities $(x, y)$ results in a set of numbers $\left(x_{0}, y_{0}, \chi_{0}^{2}\right),\left(x_{1}, y_{1}, \chi_{1}^{2}\right), \ldots,\left(x_{n}, y_{n}, \chi_{n}^{2}\right)$ after $(n+1)$ variations of the analysis procedure, with $\chi_{0}^{2}=\min _{i=0, n} \chi_{i}^{2}$. First a systematic correlation coefficient is calculated according to

$$
\rho_{x, y}^{\text {syst }}=\frac{\sum_{i=1}^{n}\left(x_{0}-x_{i}\right)\left(y_{0}-y_{i}\right)}{\sqrt{\left(\sum_{i=1}^{n}\left(x_{0}-x_{i}\right)^{2}\right)\left(\sum_{i=1}^{n}\left(y_{0}-y_{i}\right)^{2}\right)}} .
$$

Then the elements of the systematic covariance matrix are defined as

$$
\sqrt{\sigma_{x, x}^{\text {syst }}}=C \max _{i=1, n}\left(\frac{\Delta x_{i}}{\sqrt{\Delta \chi_{i}^{2}}}\right) \quad, \quad \sigma_{x, y}^{\text {syst }}=\rho_{x, y}^{\text {syst }} \sqrt{\sigma_{x, x}^{\text {syst }}} \sqrt{\sigma_{y, y}^{\text {syst }}}
$$

with

$$
\Delta x_{i}=\left|x_{0}-x_{i}\right| \quad, \quad \Delta \chi_{i}^{2}=\max \left(1,\left|\chi_{0}^{2}-\chi_{i}^{2}\right|\right) \quad, \quad C=\max \left(1, \sqrt{\chi_{0}^{2} / N_{d o f}}\right),
$$

and $\sigma_{y, y}^{\text {syst }}$ defined in analogy to $\sigma_{x, x}^{\text {syst }}$. The factor $C$ takes into account cases where the best fit gives a bad $\chi^{2}$, which does not happen in this analysis. This scheme is generalizable to any number of fit variables, and it is ensured that models giving a bad fit are properly deweighted. Of course still some unavoidable arbitrariness remains in the choice and number of variations.

\subsection{Theoretical Predictions}

As already mentioned in Section 4.3, systematic uncertainties are caused by ambiguities in the choice of matching scheme and renormalization scale for the perturbative prediction of the differential two-jet rate. In order to estimate these errors, the renormalization scale for each matching scheme was varied around the value giving the minimum $\chi^{2}$ until an increase of one unit was found. Hence there are six variations which are used in the scheme described above.

Tables 2, 3 and 4 in the Appendix contain all of the fit results under those variations. Furthermore Fig. 4 illustrates the dependence of the $\chi^{2}$ and the fit values on the renormalization scale, for both matching schemes. There is a clear preference for lower scales by the logR compared to the $\mathrm{R}$ matching scheme. This could mainly arise from the fact that in the $\log \mathrm{R}$ scheme the lowest order coefficients $A(y), B(y)$ are exponentiated, giving subleading contributions in third and higher orders. These are not present in the case of the $\mathrm{R}$ scheme.

The following systematic uncertainties are found : 
Fit $\mathrm{A}: \quad \Delta^{t h} \bar{\alpha}_{s}\left(\mathrm{M}_{\mathrm{Z}}\right)=0.0006, \quad \Delta^{t h} f_{A}=0.07, \quad \Delta^{t h} f_{T}=0.23$,

$$
\rho_{\bar{\alpha}_{s}, f_{A}}^{t h}=-0.99, \quad \rho_{\bar{\alpha}_{s}, f_{T}}^{t h}=0.90, \quad \rho_{f_{A}, f_{T}}^{t h}=-0.94 \text {. }
$$

Fit B : $\quad \Delta^{t h} \alpha_{s}\left(\mathrm{M}_{\mathrm{Z}}\right)=0.0026, \quad \Delta^{t h} n_{f}=1.01, \quad \rho_{\alpha_{s}, n_{f}}^{t h}=0.94$

Fit $\mathrm{C}: \quad \Delta^{t h} \alpha_{s}\left(\mathrm{M}_{\mathrm{Z}}\right)=0.0037$.

\subsection{Hadronization Corrections}

A large set of different models have been analysed in order to estimate the contribution to the systematics induced by a special choice of the hadronization correction. As two different $\mathrm{MC}$ models are used to calculate the correction (cf. Section 4.3.1), variations of both models have been considered separately.

Variations of the corrections for the differential two-jet rate are ( $P S=$ Parton Shower, $\mathrm{ME}=$ Matrix Element option, $\mathrm{SF}=$ String Fragmentation, $\mathrm{CF}=$ Cluster Fragmentation) :

- JETSET 7.405 PS+SF, Bose-Einstein correlations included but no retuning of the model parameters,

- HERWIG 5.8 PS+CF, tuned parameters,

- ARIADNE 4.05 PS+SF, tuned parameters.

Variations of the corrections for the four-jet angular variables, not used in Fit C, are :

- JETSET 7.405 ME+SF, tuned parameters,

- JETSET 7.405 ME+SF, only variables such as $p_{\perp}^{\text {out }}$ and tails of event-shape distributions used for parameter tuning,

- HERWIG 5.9A ME+CF, hadronization parameters changed (effective gluon mass set to $0.750 \mathrm{GeV}$ instead of $0.668 \mathrm{GeV}$; maximum cluster mass set to $3.40 \mathrm{GeV}$ instead of 3.65 $\mathrm{GeV})$

The results are :

$$
\text { Fit A } \begin{array}{rlrl}
\Delta^{h a} \bar{\alpha}_{s}\left(\mathrm{M}_{\mathrm{Z}}\right) & =0.0005, & \Delta^{h a} f_{A}=0.03, & \Delta^{h a} f_{T}=0.11 \\
\rho_{\bar{\alpha}_{s}, f_{A}}^{h a} & =0.15, \quad \rho_{\bar{\alpha}_{s}, f_{T}}^{h a}=0.69, \quad \rho_{f_{A}, f_{T}}^{h a}=0.82 .
\end{array}
$$

Fit B : $\quad \Delta^{h a} \alpha_{s}\left(\mathrm{M}_{\mathrm{Z}}\right)=0.0025, \quad \Delta^{h a} n_{f}=0.45, \quad \rho_{\alpha_{s}, n_{f}}^{h a}=0.99$

Fit $\mathrm{C}: \quad \Delta^{h a} \alpha_{s}\left(\mathrm{M}_{\mathrm{Z}}\right)=0.0008$.

As can be observed from Tables 2, 3 and 4, the variations concerning the two-jet rate have small impact, whereas the main differences arise from the use of the matrix element option in JETSET. It has been found that an increase in the intrinsic $y_{c u t}$ is accompanied by an increase in the fitted 
values of $f_{A}$ and $f_{T}$, which could explain the high values found for this model, where the intrinsic

$y_{\text {cut }}$ is rather high as mentioned in Section 4.3.1. However, using this model results in a very bad $\chi^{2}$, which suppresses its contribution to the final error. It is remarkable that the drastic change in fragmentation parameters in HERWIG 5.9A causes only a small change in the fit values. Although this variation gives the smallest $\chi^{2}$, it is not used for the nominal fit, the main reason being that it is based on smaller statistics.

In agreement with previous studies [11], the hadronization error for the case of the Fit C analysis is small. This seems to be in agreement with results of recent theoretical developments on power law corrections to event shapes, which indicate that the differential two-jet rate shows a stronger suppression of non-perturbative contributions than other variables [24].

\subsection{Detector Simulation}

A further source of systematic uncertainties are imperfections of the detector simulation which is used to estimate the effects of finite detector resolution and acceptance. The standard method is to vary the selection cuts (described in Section 4.2), especially on quantities which are known to show defects in the simulation, such as the low energy tails of neutral particles. The list of variations studied is :

- analysis with charged particle tracks only (without the cut on the total multiplicity),

- removal of the cut on the neutral energy,

- removal of the cut on the total multiplicity,

- removal of the cut on the polar angle of the sphericity axis.

A breakdown of the fit results under these variations can be found in Tables 2,3 and 4 . The resulting systematic errors are :

$$
\text { Fit A : } \begin{aligned}
\Delta^{d e} \bar{\alpha}_{s}\left(\mathrm{M}_{\mathrm{Z}}\right) & =0.0003, \quad \Delta^{d e} f_{A}=0.07, \quad \Delta^{d e} f_{T}=0.10 \\
\rho_{\bar{\alpha}_{s}, f_{A}}^{d e} & =-0.31, \quad \rho_{\bar{\alpha}_{s}, f_{T}}^{d e}=0.20, \quad \rho_{f_{A}, f_{T}}^{d e}=0.86 .
\end{aligned}
$$

Fit B : $\quad \Delta^{d e} \alpha_{s}\left(\mathrm{M}_{\mathrm{Z}}\right)=0.0016, \quad \Delta^{d e} n_{f}=0.27, \quad \rho_{\alpha_{s}, n_{f}}^{d e}=0.72$.

Fit $\mathrm{C}: \Delta^{d e} \alpha_{s}\left(\mathrm{M}_{\mathrm{Z}}\right)=0.0006$

The analysis based on charged particle tracks alone shows differences in the final fit results. In the case of the Fit $\mathrm{C}$ analysis a detailed study indicates that the poor description of the charged inclusive momentum distribution out of the event plane $p_{\perp}^{\text {out }}$ by the standard MC models [20] might be at the origin of those deviations. Removing tracks from the high tail of this distribution $\left(p_{\perp}^{\text {out }}>1 \mathrm{GeV} / c\right)$ brings the fit results again in agreement with the nominal analysis. 


\subsection{Mass Effects}

The mass of the b quark is expected to influence the cross sections, because of dynamical and mainly phase space effects, since the $y_{\text {cut }}$ value applied is not large enough to ensure that all hard four-jet events with b quarks in the final state are resolved. Unfortunately, in contrast to Born level calculations at first and second order [25], a full second order calculation (with loop corrections) for massive quarks is not available. This would be necessary to estimate consistently mass effects in this analysis. However, in a previous analysis [26] it has been found that mass effects have negligible impact on the shape of the $-\ln y_{3}$ distribution, at least over the fit range used in this analysis.

Because of this observation, in Fit A the study of mass effects can be restricted to the four angular variables. Those were calculated with and without quark masses $\left(m_{u d s}=0 \mathrm{GeV} / c^{2}, m_{c}=\right.$ $\left.1.5 \mathrm{GeV} / c^{2}, m_{b}=5 \mathrm{GeV} / c^{2}\right)$ using the matrix elements from Ref. [25], and subsequently fitted using the massless ERT formula (8). The $-\ln y_{3}$ distribution essentially fixes $b_{0}$. While considering only the angular variables, this can be taken into account by fitting only for $f_{T}$ and calculating $f_{A}$ from $b_{0}$ according to $f_{A}=6 / 11\left(b_{0}+2 / 3 f_{T}\right)$. Here $b_{0}$ is fixed to 2.90 as found in the nominal analysis with the $\mathrm{R}$ matching scheme. When fitting the massive matrix elements, an upwards shift in $f_{T}$ of 0.25 is observed with respect to the massless case. This arises from the fact that the change of the cross sections due to phase-space reduction is not uniform over the range of the angular distributions, and is different for q⿳亠口gg final states compared to four-quark final states. A similar result was obtained in an analysis by OPAL [14]. From this it is concluded that mass

effects can be taken account of by the replacement $n_{f}^{e f f}=n_{f}+0.67$ in the four-jet matrix elements. From the statistical errors and correlations it follows that the change in $f_{T}$ under inclusion of mass effects implies a change in $f_{A}$ by -0.07 and in $\bar{\alpha}_{s}$ by -0.0002 . The difference of the results with and without mass effects was added quadratically to the systematic error.

In Fit $\mathrm{B}$ the main sensitivity comes from the $-\ln y_{3}$ distribution, and the angular variables only help to restrict the systematic uncertainties. Nevertheless, in order to take into account mass effects in this case, the fit was repeated by replacing $n_{f}$ in Eq. (8) with $n_{f}^{e f f}=n_{f}+0.67$, as suggested by the findings for mass effects on Fit A, and leaving the formula for the differential two-jet rate and the running of $\alpha_{s}$ unchanged. Only small shifts of -0.03 for $n_{f}$ and -0.0002 for $\alpha_{s}\left(\mathrm{M}_{\mathrm{Z}}\right)$ are found. Again the difference of the results when including mass effects was taken as an additional error contribution, which however has negligible impact on the final error.

\subsection{Further Systematic Checks}

A stringent test of the influence of hadronization has been performed by repeating the fit without hadronization corrections. The deviations for $\bar{\alpha}_{s}$ and $f_{A}$ are very small and covered by the systematic error, and the deviations for $f_{T}$ are not larger than $40 \%$ with respect to the nominal values, which is reassuring. As the hadronization models do not include contributions from gluinos, a rough simulation of the effects of a possible $R^{0}$ hadron (a gluon-gluino bound state) [5] has been attempted using the JETSET string model. The production of strange quarks and di-quarks and the width of the transverse momentum distribution of particles generated from the string has been increased, and the hadronization corrections were recomputed for the differential two-jet rate, which gives the main sensitivity for Fit B. Negligible effects on the final results are found. In 
addition, one can expect that hadronization models, which could bring the data into agreement with the light gluino hypothesis, would drastically worsen the overall description of the properties of hadronic $\mathrm{Z}$ decays.

Further it has been checked that the fit is not strongly biased towards the QCD colour factors used in the MC simulations by changing the colour factors in the models to values far from QCD and recalculating all corrections. These checks gave satisfactory results, in the sense that the observed changes are covered by the systematic errors.

Variations of the fit ranges by \pm one bin in all distributions were found to have only a small impact on the fit results, which at most changed by half the statistical error.

Finally it is worth noting that first results of a calculation of the next-to-leading order corrections (neglecting terms of $\mathcal{O}\left(1 / N_{C}^{2}\right)$ ) to the angular variables [27] indicate that these corrections have a large impact on the four-jet rate, but very small influence on the shape of the angular distributions. Since this analysis is only sensitive to the shape, the inclusion of higher order corrections would therefore lead to minor changes in the final results.

The final systematic errors and correlations are obtained by summing the covariance matrices of the individual contributions to the systematics. They are given below. The much smaller correlation between $f_{A}$ and $f_{T}$ compared to the purely statistical one stems from the anticorrelation induced by the theoretical uncertainties.

$$
\text { Fit A : } \begin{aligned}
\Delta^{s y} \bar{\alpha}_{s}\left(\mathrm{M}_{\mathrm{Z}}\right) & =0.0009, \quad \Delta^{s y} f_{A}=0.13, \quad \Delta^{s y} f_{T}=0.35, \\
\rho_{\bar{\alpha}_{s}, f_{A}}^{s y} & =-0.30, \quad \rho_{\bar{\alpha}_{s}, f_{T}}^{s y}=0.69, \quad \rho_{f_{A}, f_{T}}^{s y}=0.21 .
\end{aligned}
$$

Fit B : $\quad \Delta^{s y} \alpha_{s}\left(\mathrm{M}_{\mathrm{Z}}\right)=0.0040, \quad \Delta^{s y} n_{f}=1.15, \quad \rho_{\alpha_{s}, n_{f}}^{s y}=0.87$.

Fit $\mathrm{C}: \Delta^{s y} \alpha_{s}\left(\mathrm{M}_{\mathrm{Z}}\right)=0.0038$.

\section{Results and Discussion}

As final results the weighted means (using $\left(\chi^{2} / N_{d o f}\right)^{-1}$ as weights) of the fit values obtained in the nominal analysis with the two matching schemes, together with the sum of statistical and systematic covariance matrices, are (under inclusion of mass effects) :

$$
\begin{gathered}
\text { Fit A : } \quad \begin{array}{r}
\bar{\alpha}_{s}\left(\mathrm{M}_{\mathrm{Z}}\right)=0.0244 \pm 0.0003(\text { stat }) \pm 0.0009 \text { (syst) } \\
f_{A}=2.20 \pm 0.09 \text { (stat) } \pm 0.13 \text { (syst) } \\
f_{T}=1.63 \pm 0.29(\text { stat }) \pm 0.35 \text { (syst) } \\
n_{f}^{\text {eff }}=5.67 \rightarrow \frac{T_{F}}{C_{F}}=0.29 \pm 0.05 \text { (stat) } \pm 0.06 \text { (syst) } \\
\rho_{\bar{\alpha}_{s}, f_{A}}=-0.15 \quad, \quad \rho_{\bar{\alpha}_{s}, f_{T}}=0.65 \quad, \quad \rho_{f_{A}, f_{T}}=0.47
\end{array}
\end{gathered}
$$


Fit $\mathrm{B}: \quad \alpha_{s}\left(\mathrm{M}_{\mathrm{Z}}\right)=0.1162 \pm 0.0012$ (stat) \pm 0.0040 (syst)

$$
\begin{gathered}
n_{f}=4.24 \pm 0.29(\text { stat }) \pm 1.15 \text { (syst) } \\
\rho_{\alpha_{s}, n_{f}}=0.88
\end{gathered}
$$

Fit $\mathrm{C}: \quad \alpha_{s}\left(\mathrm{M}_{\mathrm{Z}}\right)=0.1195 \pm 0.0002$ (stat) \pm 0.0038 (syst)

The summation of the systematic covariance matrices with the purely statistical one leads to a reduction of the correlations, at the price of larger uncertainties. In Fig. 5 it can be observed that the measurement of the colour factors is in agreement with the expectations from QCD $\left(C_{A} / C_{F}=2.25, T_{F} / C_{F}=0.375\right)$. Furthermore it is in agreement with previous measurements by ALEPH [20] and by other collaborations [12, 14]. Here a more general approach is adopted, as the coupling constant is fitted as well. Figures 6 and 7 show that the measured coupling constant, although determined model independently, is consistent with the world average value of $\alpha_{s}\left(\mathrm{M}_{\mathrm{Z}}\right)=0.118 \pm 0.003[28]$.

The measurement of $\alpha_{s}\left(\mathrm{M}_{\mathrm{Z}}\right)\left(\mathrm{QCD}, n_{f}=5\right)$ represents a very accurate determination of the strong coupling constant via event shapes in $e^{+} e^{-}$annihilation and agrees with the world average value. However, this measurement is mainly intended to serve as a cross check for the more general analysis, and indeed perfect consistency is found. An ALEPH measurement of $\alpha_{s}\left(\mathrm{M}_{\mathrm{Z}}\right)$, including new data at higher centre-of-mass energies and new theoretical input regarding powerlaw corrections to event shapes, is under way.

\subsection{A Limit on the Light Gluino}

Supersymmetric models predict the existence of a fermionic partner of the gluon, called gluino. Being a Majorana fermion and transforming under the adjoint representation of SU(3), at leading order the effects of a light gluino are taken account of by simply adding three fermionic degrees of freedom. Such a gluino would give loop corrections to the gluon propagator, thus changing the running of $\alpha_{s}$, and it would lead to additional four-parton final states via gluon splitting into a pair of gluinos. These gluinos would then be confined into neutral $R^{0}$ hadrons [5]. Because of the rescaling of jet energies, this analysis is not sensitive to the details of gluino hadronization and possible decays.

The lowest mass scale entering into the prediction for the differential two-jet rate is the upper limit of the fit range in Fit $\mathrm{B}$, namely $k_{\perp} \approx 10 \mathrm{GeV}$. Thus the replacement $n_{f}=5 \rightarrow n_{f}=8$ in the expression for the running of $\alpha_{s}$ would be valid for gluino masses up to $10 \mathrm{GeV} / c^{2}$, which is not compatible with the data. A more conservative limit on the gluino mass is found by estimating the effect on the angular variables. Again employing the matrix elements for $q \bar{q} q^{\prime} \bar{q}^{\prime}$ with inclusion of quark masses [25], it has been found that the effect of a finite quark mass on the cross section of the gluon splitting process can be described by the simple phase space reduction factor $\sqrt{1-4 m_{q}^{2} /\left(s y_{c u t}^{J}\right)}$. As the invariant mass of the decaying system is of relevance, the Jade definition of the cut-off value has to be taken here instead of the Durham definition used throughout the analysis. This corresponds to a substitution of $y_{\text {cut }}=0.008$ with $y_{\text {cut }}^{J}=0.032$. For example, it has been checked that this formula reproduces correctly an overall reduction of the cross section of $20 \%$ found with the massive matrix elements for a quark mass of $5 \mathrm{GeV} / c^{2}$. 
From Figs. 5 and 7 it is clear that even the model independent analysis (Fit A) excludes the existence of a light gluino at very high confidence level (CL). From the results of Fit B (Fig. 8) an upper limit on the excess in $n_{f}$ above its expectation of five of $\Delta n_{f}<1.9$ at $95 \%$ CL is found. From this a lower limit on the gluino mass is obtained according to

$$
\Delta n_{f}=3 \sqrt{1-\frac{4 m_{\tilde{g}}^{2}}{s y_{\text {cut }}^{J}}}<1.9
$$

which translates into $m_{\tilde{g}}>6.3 \mathrm{GeV} / c^{2}$ at $95 \% \mathrm{CL}$.

These results are in agreement with the analysis of the running of $\alpha_{s}$ in Ref. [29], which excludes the gluino for masses below $3.8 \mathrm{GeV} / c^{2}$ at $90 \%$ CL. Recently a study of the running of $\alpha_{s}$ has been suggested in Ref. [30], leading to similar conclusions. In Ref. [31] a reanalysis of published colour factor measurements has been proposed in order to set a limit on the gluino mass.

\section{Conclusions}

A precise test of perturbative QCD has been performed by comparing theoretical predictions of four-jet angular variables and the differential two-jet rate to ALEPH data taken from 1992 to 1995. Colour factors in agreement with QCD have been measured,

$$
\begin{aligned}
& \frac{C_{A}}{C_{F}}=2.20 \pm 0.09(\text { stat }) \pm 0.13(\text { syst }), \\
& \frac{T_{F}}{C_{F}}=0.29 \pm 0.05(\text { stat }) \pm 0.06(\text { syst }),
\end{aligned}
$$

together with a precise determination of $\alpha_{s}\left(\mathrm{M}_{\mathrm{Z}}\right)$. The existence of a gluino in the mass range below $6.3 \mathrm{GeV} / c^{2}$ is excluded at $95 \%$ confidence level.

\section{Acknowledgements}

We thank our colleagues from the accelerator divisions for the successful operation of the LEP machine and the engineers and technical staff in all our institutions for their contribution to the good performance of ALEPH. Those of us from non-member states thank CERN for its hospitality. 


\section{A Appendix}

In the following listings of fit results under all variations of the analysis are given. If not indicated otherwise, the $\mathrm{R}$ matching scheme is used at the renormalization scale with minimal $\chi^{2}$, together with the data selection and hadronization corrections as described in sections 4.2 and 4.3.1. As hardly any variations of the statistical correlations could be observed, they are not given here.

The abbreviations are : JS = JETSET 7.405, HW58 = HERWIG 5.8, HW59= HERWIG 5.9A, AR = ARIADNE 4.05, PS = Parton Shower, ME = Matrix Element option, $\mathrm{SF}$ $=$ String Fragmentation, $\mathrm{CF}=$ Cluster Fragmentation, $\mathrm{BE}=$ Bose-Einstein correlations, $\mathrm{OUT}=$ Out quantities used for model tuning (cf. section 5.3), HPC = hadronization parameters changed, $\ln f=\ln \mu^{2} / s, \mathrm{CHG}=$ charged tracks, NEU $=$ neutral tracks, $E_{\text {neu }}=$ neutral energy, $N_{\text {tot }}=$ total multiplicity, nominal $=$ nominal analysis (cf. section 5.1).

\begin{tabular}{|l|c|c|c|c|}
\hline Variations & $\bar{\alpha}_{s}\left(\mathrm{M}_{\mathrm{Z}}\right)$ & $f_{A}$ & $f_{T}$ & $\chi^{2}$ \\
\hline \hline \multicolumn{4}{|c|}{ Theoretical Prediction } \\
\hline \hline nominal $: \operatorname{logR}, \ln f=-1.1$ & 0.0244 & 2.31 & 1.48 & 78.1 \\
\hline $\operatorname{logR}, \ln f=-1.7$ & 0.0250 & 2.25 & 1.51 & 79.3 \\
$\operatorname{logR}, \ln f=-0.3$ & 0.0240 & 2.36 & 1.39 & 79.1 \\
$\mathrm{R}, \ln f=-0.55$ & 0.0256 & 2.16 & 1.95 & 82.6 \\
$\mathrm{R}, \ln f=0.0$ & 0.0249 & 2.23 & 1.78 & 81.6 \\
$\mathrm{R}, \ln f=0.85$ & 0.0242 & 2.34 & 1.49 & 82.6 \\
\hline \hline \multicolumn{4}{|c|}{} \\
\hline \hline nominal $:$ HW59 ME+CF, JS PS+SF & 0.0249 & 2.23 & 1.78 & 81.6 \\
\hline HW59 ME+CF, JS PS+SF, BE & 0.0251 & 2.23 & 1.83 & 83.2 \\
HW59 ME+CF, HW58 PS+CF & 0.0264 & 2.17 & 2.10 & 90.8 \\
HW59 ME+CF, AR PS+SF & 0.0252 & 2.23 & 1.85 & 83.2 \\
JS ME+SF, JS PS+SF & 0.0254 & 2.41 & 2.40 & 124.5 \\
JS ME+SF OUT, JS PS+SF & 0.0253 & 2.40 & 2.36 & 115.2 \\
HW59 ME+CF HPC, JS PS+SF & 0.0250 & 2.27 & 1.90 & 74.8 \\
\hline \hline \multicolumn{4}{|c|}{ Detector Corrections } \\
\hline \hline nominal $:$ CHG+NEU & 0.0249 & 2.23 & 1.78 & 81.6 \\
\hline CHG only & 0.0248 & 2.14 & 1.39 & 116.5 \\
no cut on $E_{n e u}$ & 0.0247 & 2.25 & 1.68 & 95.6 \\
no cut on $N_{\text {tot }}$ & 0.0249 & 2.24 & 1.81 & 81.9 \\
no cut on $\theta_{\text {sph }}$ & 0.0246 & 2.30 & 1.88 & 82.6 \\
\hline
\end{tabular}

Table 2: Variations of the Fit A analysis, $N_{d o f}=72$. 


\begin{tabular}{|c|c|c|c|}
\hline Variations & $\alpha_{s}\left(\mathrm{M}_{\mathrm{Z}}\right)$ & $n_{f}$ & $\chi^{2}$ \\
\hline \multicolumn{4}{|c|}{ Theoretical Prediction } \\
\hline nominal : $\log \mathrm{R}, \ln f=-1.3$ & 0.1154 & 3.68 & $\overline{78.5}$ \\
\hline $\log R, \ln f=-1.8$ & 0.1182 & 4.08 & 79.8 \\
\hline $\log R, \ln f=-0.6$ & 0.1130 & 3.09 & 79.6 \\
\hline $\mathrm{R}, \ln f=-0.5$ & 0.1210 & 5.81 & 83.3 \\
\hline $\mathrm{R}, \ln f=0.0$ & 0.1175 & 4.88 & 81.6 \\
\hline $\mathrm{R}, \ln f=0.7$ & 0.1141 & 3.57 & 83.0 \\
\hline \multicolumn{4}{|c|}{ Hadronization Corrections } \\
\hline nominal : HW59 ME+CF, JS PS+SF & 0.1175 & 4.88 & 81.6 \\
\hline HW59 ME+CF, JS PS+SF, BE & 0.1184 & 5.04 & 83.3 \\
\hline HW59 ME+CF, HW58 PS+CF & 0.1250 & 6.21 & 91.6 \\
\hline HW59 ME+CF, AR PS+SF & 0.1191 & 5.12 & 83.3 \\
\hline JS ME+SF, JS PS+SF & 0.1186 & 5.15 & 128.2 \\
\hline JS ME+SF OUT, JS PS+SF & 0.1184 & 5.12 & 118.8 \\
\hline HW59 ME+CF HPC, JS PS+SF & 0.1175 & 4.91 & 74.9 \\
\hline \multicolumn{4}{|l|}{$\begin{array}{l}\text { Detector Corrections } \\
\end{array}$} \\
\hline nominal : CHG+NEU & 0.1175 & 4.88 & 81.6 \\
\hline CHG only & 0.1178 & 4.59 & 117.4 \\
\hline no cut on $E_{\text {neu }}$ & 0.1162 & 4.51 & 96.0 \\
\hline no cut on $N_{\text {tot }}$ & 0.1173 & 4.91 & 81.9 \\
\hline no cut on $\theta_{s p h}$ & 0.1158 & 4.61 & 82.9 \\
\hline
\end{tabular}

Table 3: Variations of the Fit B analysis, $N_{\text {dof }}=73$.

\begin{tabular}{|l|c|c|}
\hline Variations & $\alpha_{s}\left(\mathrm{M}_{\mathrm{Z}}\right)$ & $\chi^{2}$ \\
\hline \hline \multicolumn{3}{|c|}{ Theoretical Prediction } \\
\hline \hline nominal : R, $\ln f=-0.25$ & 0.1176 & 5.4 \\
\hline $\mathrm{R}, \ln f=-0.55$ & 0.1174 & 6.6 \\
$\mathrm{R}, \ln f=0.1$ & 0.1180 & 6.7 \\
$\operatorname{logR}, \ln f=-2.1$ & 0.1236 & 8.1 \\
$\operatorname{logR}, \ln f=-1.76$ & 0.1220 & 6.9 \\
$\operatorname{logR}, \ln f=-1.35$ & 0.1209 & 8.0 \\
\hline \hline \multicolumn{3}{|c|}{ Hadronization Corrections } \\
\hline \hline nominal : AR PS+SF & 0.1183 & 5.1 \\
\hline JS PS+SF & 0.1176 & 5.4 \\
JS PS+SF, BE & 0.1179 & 5.7 \\
HW PS+CF & 0.1194 & 7.2 \\
\hline \hline \multicolumn{3}{|c|}{ Detector Corrections } \\
\hline \hline nominal : CHG+NEU & 0.1176 & 5.41 \\
\hline CHG only & 0.1193 & 33.76 \\
no cut on $E_{\text {neu }}$ & 0.1179 & 5.87 \\
no cut on $N_{\text {tot }}$ & 0.1174 & 5.59 \\
no cut on $\theta_{\text {sph }}$ & 0.1170 & 5.42 \\
\hline
\end{tabular}

Table 4: Variations of the Fit C analysis, $N_{d o f}=8$. 


\section{References}

[1] R.K. Ellis, D.A. Ross, and A.E. Terrano, Nucl. Phys. B178 (1981), 421.

[2] D. Decamp et al., ALEPH Collaboration, Phys. Lett. B257 (1991), 479.

[3] P.D. Acton et al., OPAL Collaboration, Z. Phys. C55 (1992), 1.

[4] S. Catani, L. Trentadue, G. Turnock, and B.R. Webber, Nucl. Phys. B407 (1993), 3.

[5] G. Farrar, Phys. Rev. D51 (1995), 3904.

[6] M. Bengtsson and P.M. Zerwas, Phys. Lett. B208 (1988), 306;

M. Bengtsson, Z. Phys. C42 (1989), 75;

J.G. Körner, G. Schierholz and J. Willrodt, Nucl. Phys. B185 (1981), 365;

O. Nachtmann and A. Reiter, Z. Phys. C16 (1982), 45;

S. Bethke, A. Richter and P.M. Zerwas, Z. Phys. C49 (1991), 59.

[7] Y.L. Dokshitzer, Workshop on Jets at LEP and HERA, Durham, 1990.

[8] S. Catani, Y.L. Dokshitzer, M. Olsson, G. Turnock, and B.R. Webber, Phys. Lett. B269 (1991), 432.

[9] N. Brown and W.J. Stirling, Z. Phys. C53 (1992), 629.

[10] G. Dissertori and M. Schmelling, Phys. Lett. B361 (1995), 167.

[11] D. Decamp et al., ALEPH Collaboration, Phys. Lett. B284 (1992), 163.

[12] P. Abreu et al., DELPHI Collaboration, Z. Phys. C59 (1993), 357.

[13] B. Adeva et al., L3 Collaboration, Phys. Lett. B248 (1990), 227.

[14] R. Akers et al., OPAL Collaboration, Z. Phys. C65 (1995), 367.

[15] D. Decamp et al., ALEPH Collaboration, Phys. Lett. B284 (1992), 151.

[16] D. Decamp et al., ALEPH Collaboration, Nucl. Instrum. Methods A294 (1990), 121.

[17] D. Buskulic et al., ALEPH Collaboration, Nucl. Instrum. Methods A360 (1995), 481.

[18] P. Nason, private communication, 1995.

[19] T. Sjöstrand, Comput. Phys. Commun. 27 (1982), 243; 28 (1983), 229;

T. Sjöstrand and M. Bengtsson, Comput. Phys. Commun. 43 (1987), 367.

[20] R. Barate et al., ALEPH Collaboration, CERN preprint CERN-PPE/96-186, submitted to Physics Reports, 1996.

[21] G. Marchesini, B.R. Webber, G. Abbiendi, I.G. Knowles, M.H. Seymour, and L. Stanco, Comput. Phys. Commun. 67 (1992), 465. 
[22] J.E. Campagne and R. Zitoun, Z. Phys. C43 (1989), 469.

[23] F. James and M. Roos, computer program MINUIT, CERN program library, writeup CERN D506, 1989.

[24] B.R. Webber, Hadronic Final States, Plenary talk at the Workshop on Deep Inelastic Scattering and QCD, Paris, April 1995.

[25] A. Ballestrero, E. Maina, and S. Moretti, Phys. Lett. B294 (1992), 425.

[26] D. Buskulic et al., ALEPH Collaboration, Phys. Lett. B355 (1995), 381.

[27] L. Dixon and A. Signer, private communication, 1996.

[28] M. Schmelling, Status of the strong coupling constant, Plenary talk at the XXVIII International Conference on High Energy Physics, Warsaw, July 1996.

[29] M. Schmelling and R. St.Denis, Phys. Lett. B329 (1994), 393.

[30] F. Csikor and Z. Fodor, CERN preprint CERN-TH/96-323, 1996.

[31] A. de Gouvêa and Hitoshi Murayama, HEP preprint hep-ph/9606449, LBL-39030, 1996. 


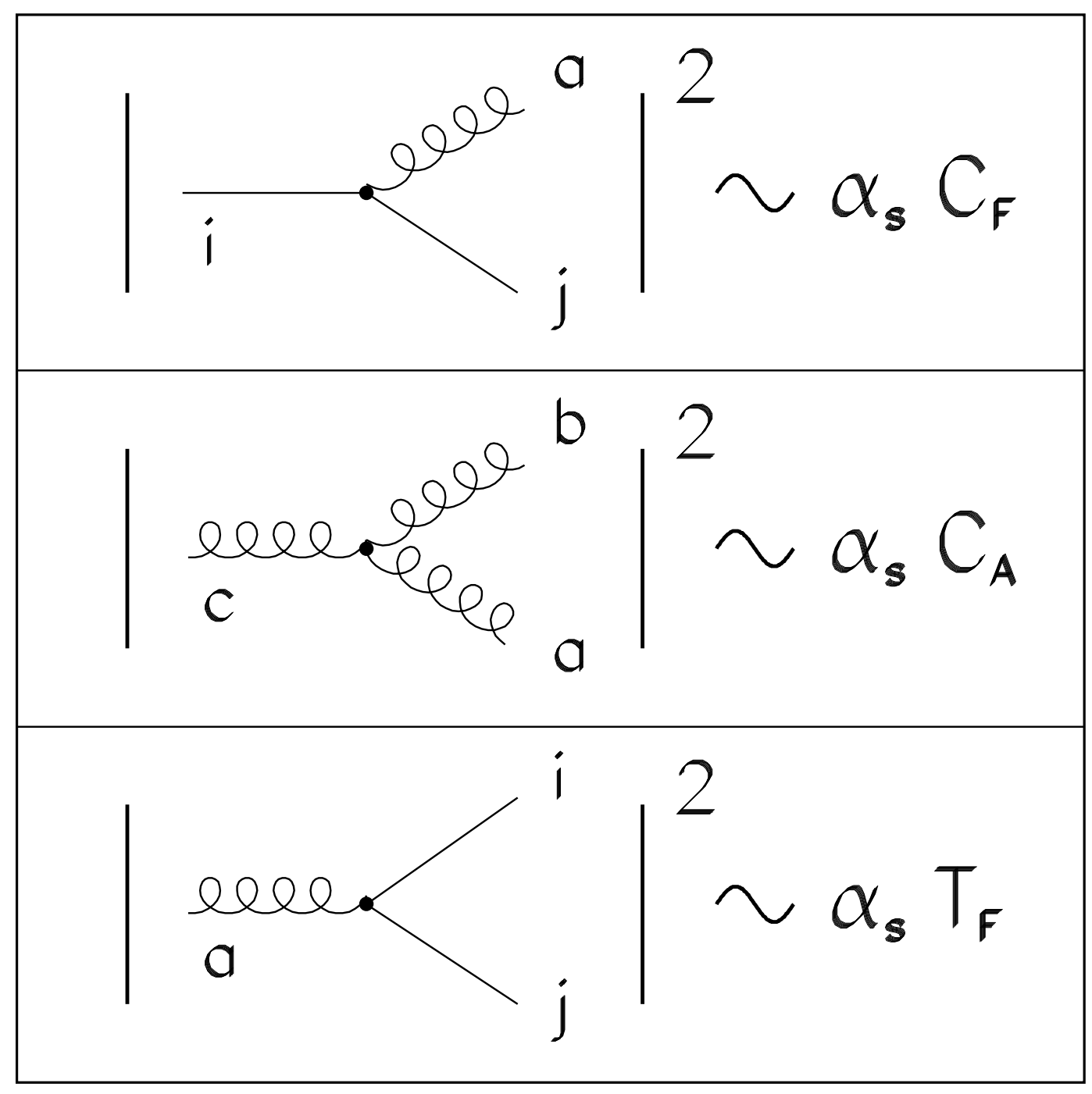

Figure 1: Relations between Casimir operators and vertices in QCD . 

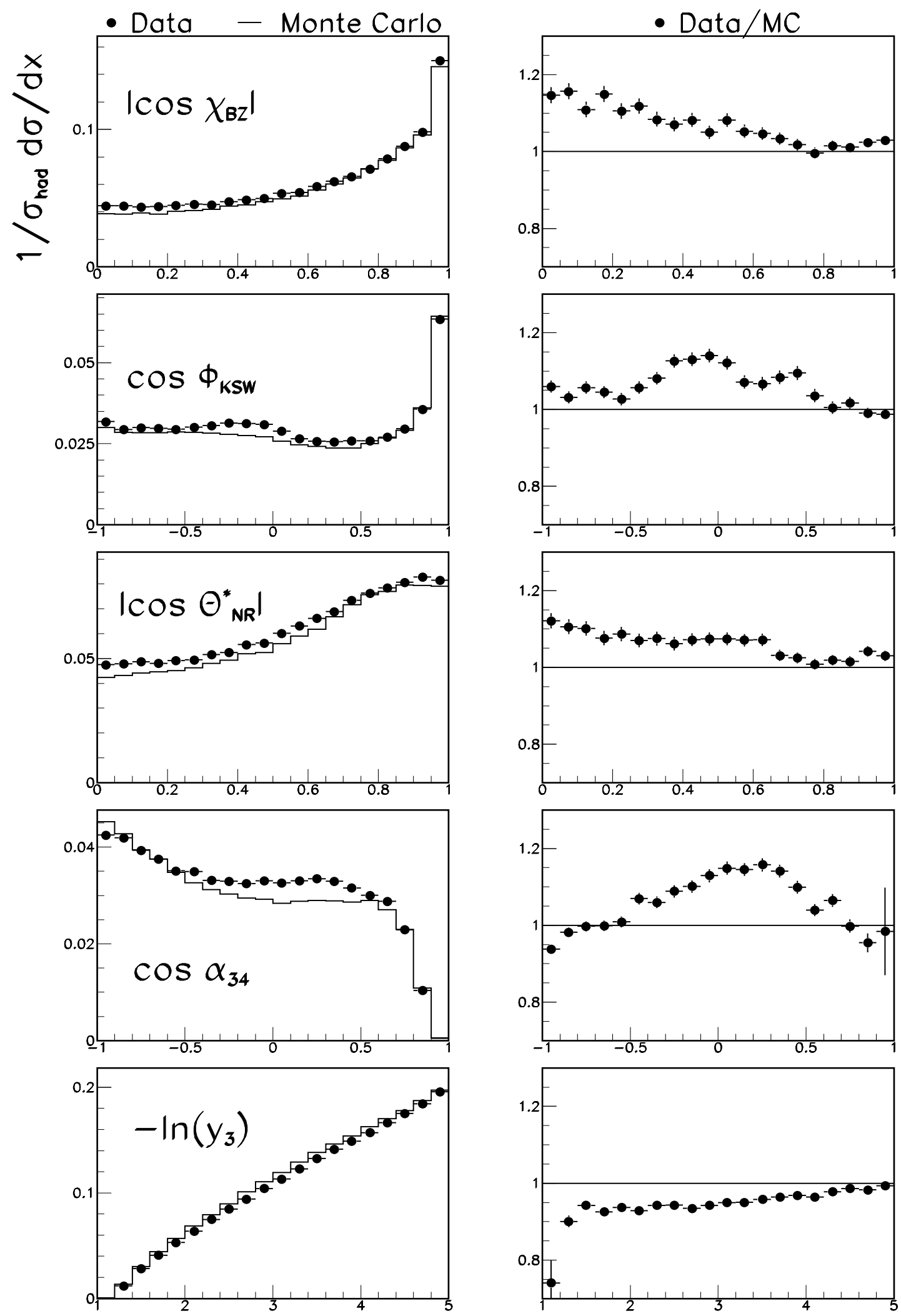

Figure 2: Comparison of ALEPH data with the full simulation (DYMU + JETSET + ALEPH detector simulation). The variables are computed at detector level . 
ALEPH
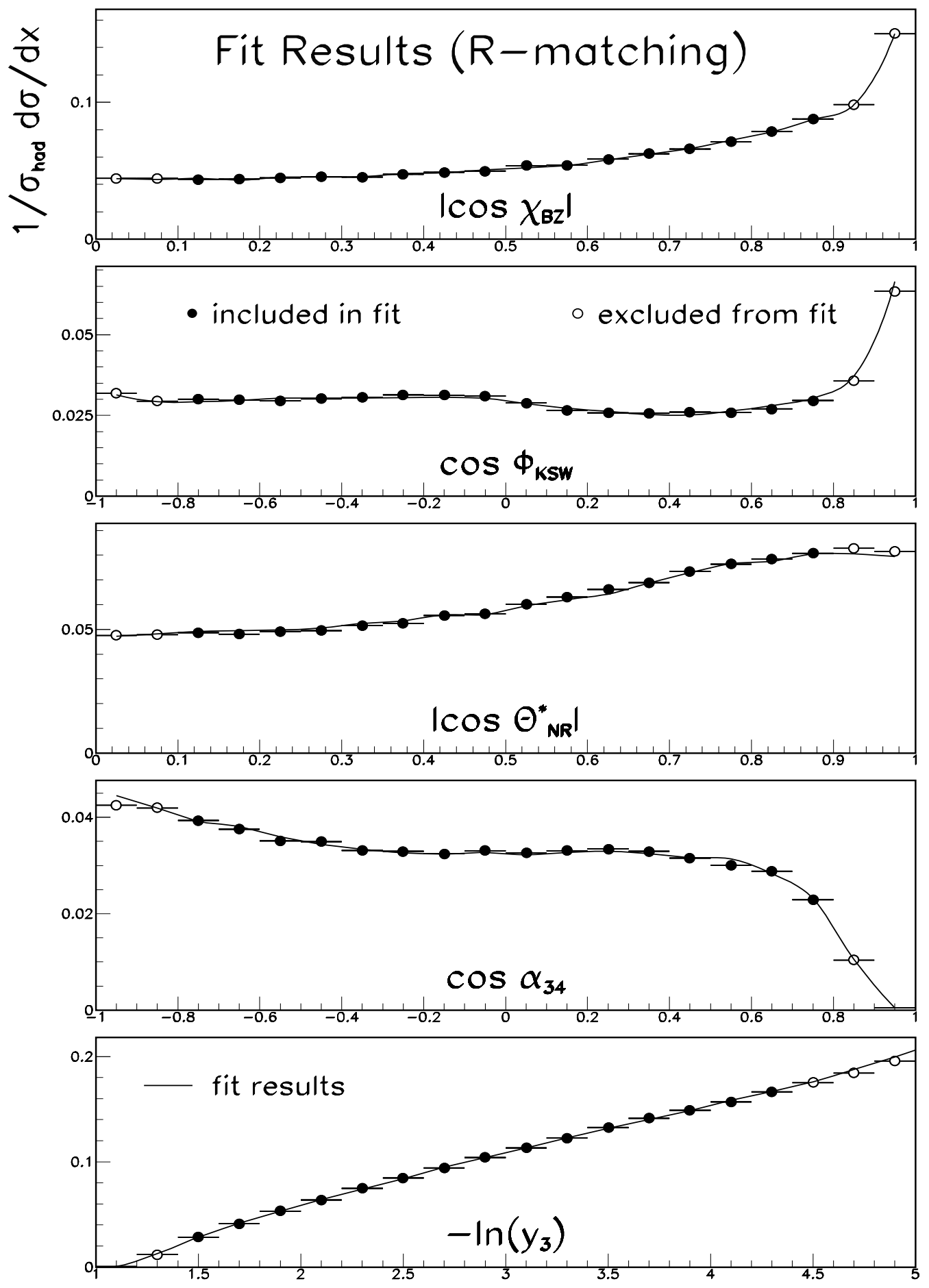

Figure 3: Fit results (fit of $\bar{\alpha}_{s}, \frac{C_{A}}{C_{F}}$ and $n_{f} \frac{T_{F}}{C_{F}}, \mathrm{R}$ matching scheme) for all five variables. The statistical error of the data is smaller than the symbol size . 


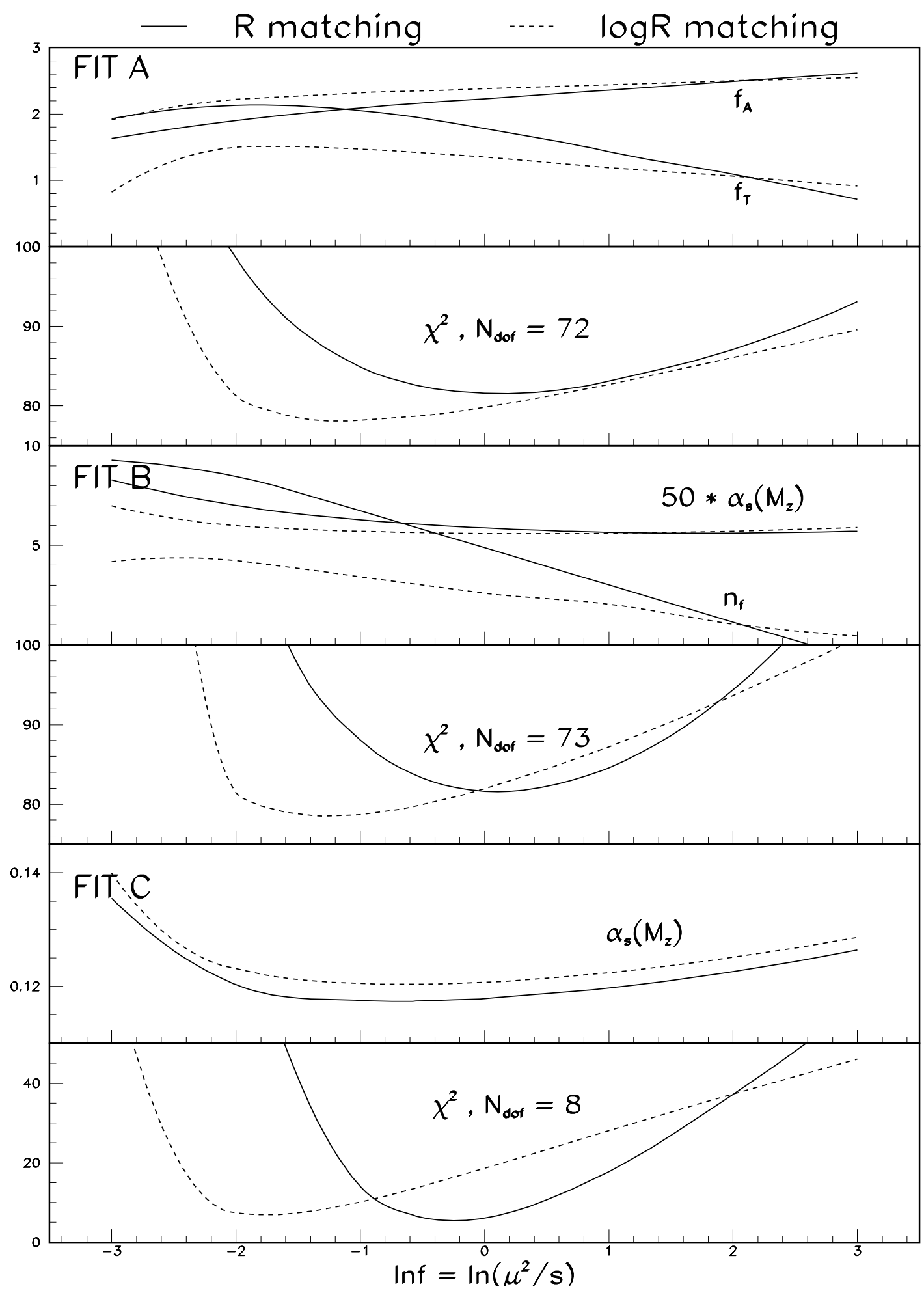

Figure 4: Dependence of the fit results on the renormalization scale $\ln f=\ln \left(\mu^{2} / s\right)$, for all three types of fits and both matching schemes. Fit A : Fit of $\bar{\alpha}_{s}, \frac{C_{A}}{C_{F}}$ and $n_{f} \frac{T_{F}}{C_{F}}$. Fit B : Fit of $\alpha_{s}$ and $n_{f}$. Fit C : Fit of $\alpha_{s}$. 


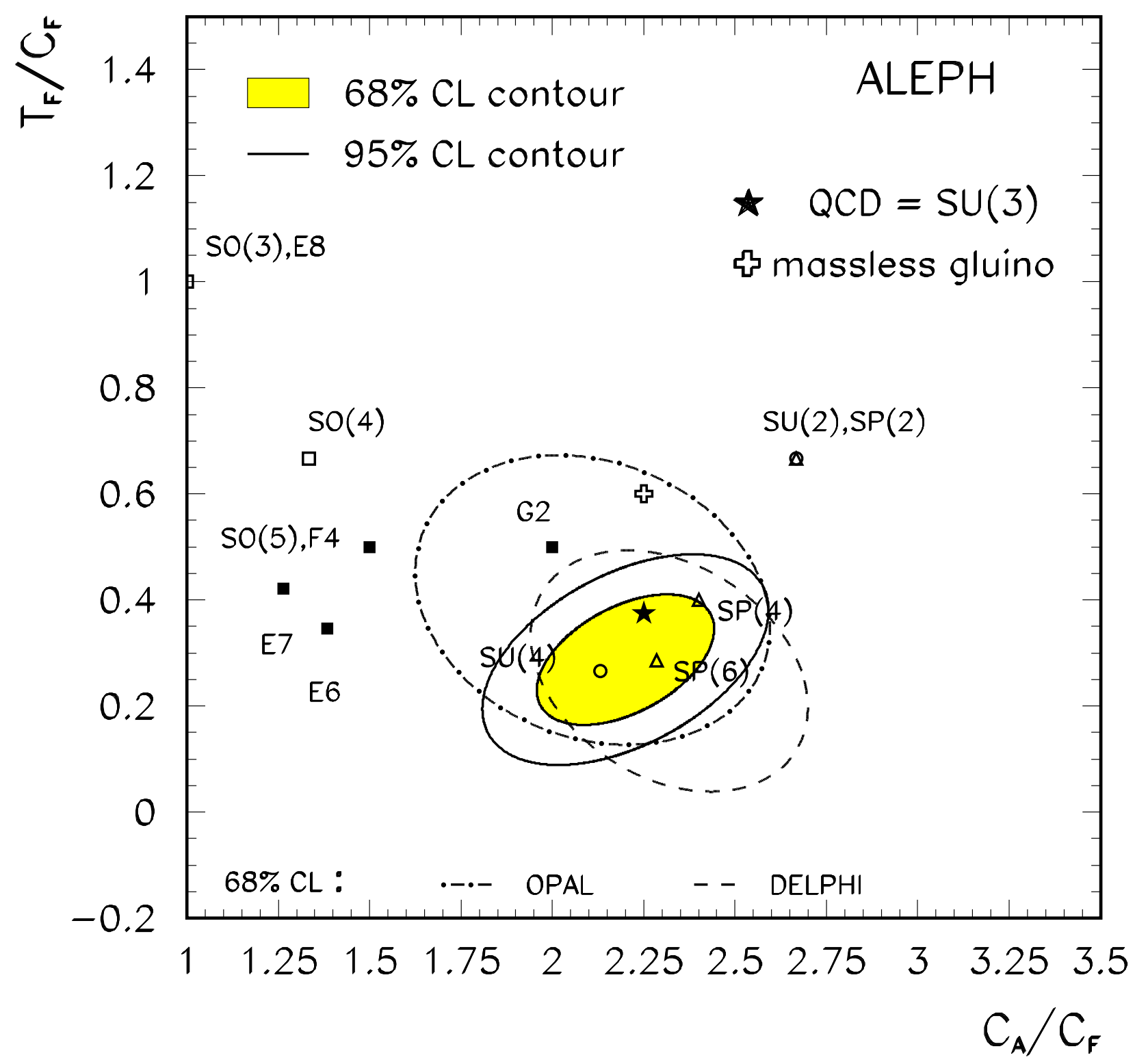

Figure 5: $68 \%$ (shaded region) and $95 \%$ (full line) confidence level contours in the $\left(\frac{C_{A}}{C_{F}}, \frac{T_{F}}{C_{F}}\right)$ plane (Fit A), calculated from statistical plus systematic error and assuming $n_{f}^{e f f}=5.67$. For comparison also the results of other measurements are given (68\% CL contours), as well as predictions for simple Lie groups . 


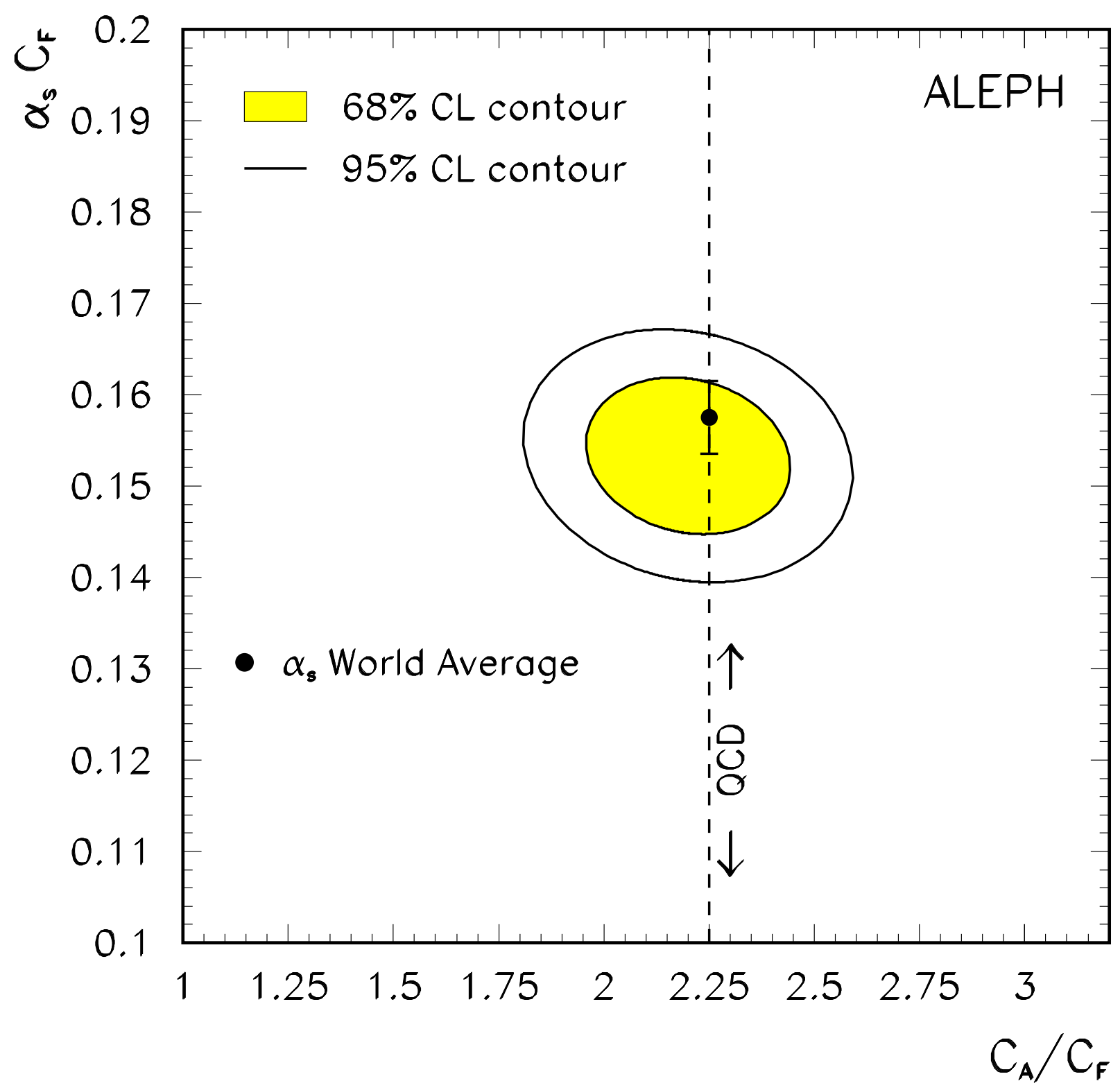

Figure 6: 68\% (shaded region) and 95\% (full line) confidence level contours in the $\left(\frac{C_{A}}{C_{F}}, \bar{\alpha}_{s}\right)$ plane (Fit A), calculated from statistical plus systematic error . 


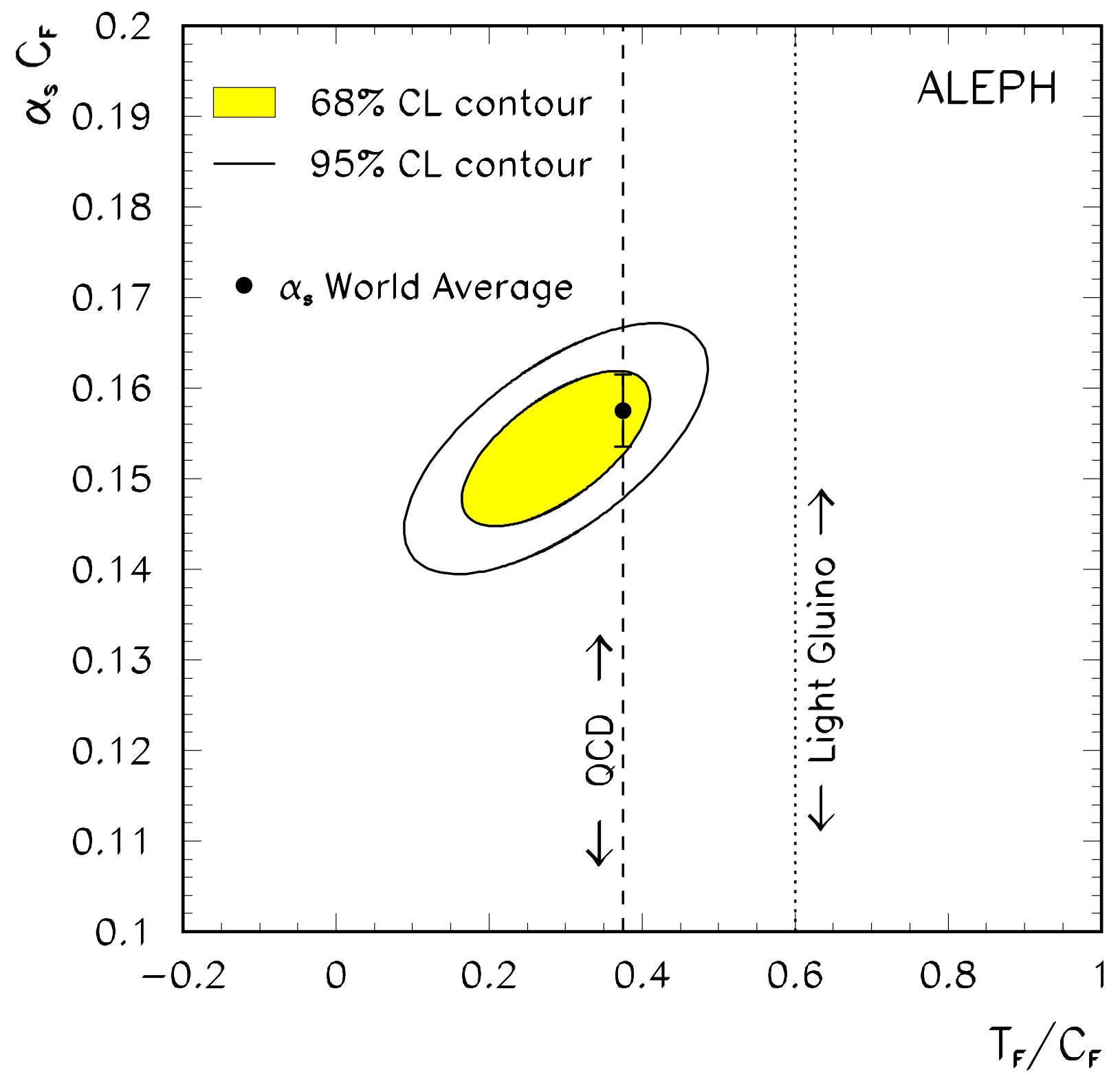

Figure 7: $68 \%$ (shaded region) and 95\% (full line) confidence level contours in the $\left(\frac{T_{F}}{C_{F}}, \bar{\alpha}_{s}\right)$ plane (Fit A), calculated from statistical plus systematic error and assuming $n_{f}^{e f f}=5.67$. The predicted values for a massless gluino are indicated by the vertical dotted line. 


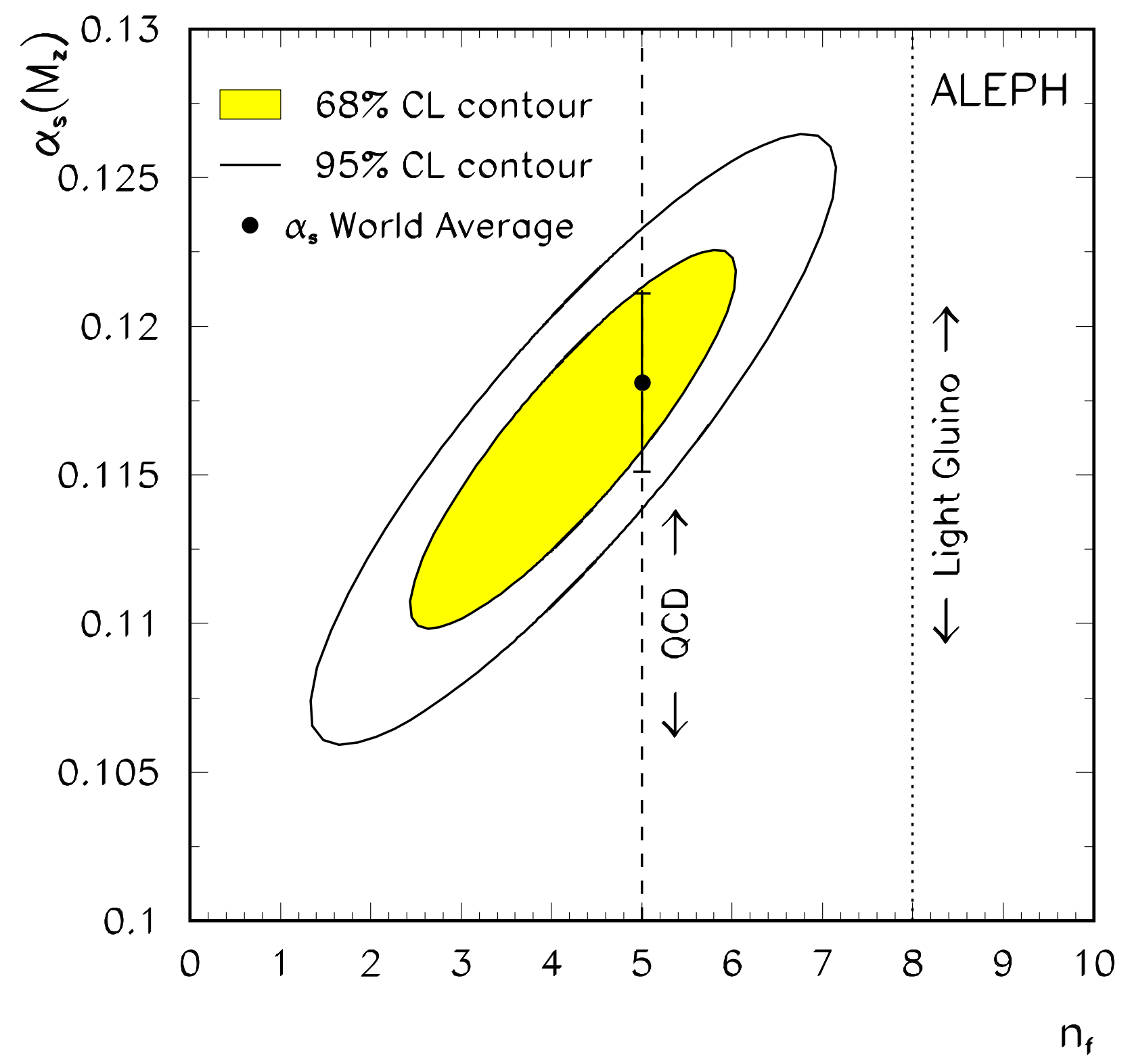

Figure 8: 68\% (shaded region) and 95\% (full line) confidence level contours in the $\left(n_{f}, \alpha_{s}\right)$ plane, calculated from statistical plus systematic error as found in Fit B (determination of $\alpha_{s}$ and $n_{f}$ ). The predicted values for a massless gluino are indicated by the vertical dotted line . 